\title{
Inflows, Outflows, and a Giant Donor in the Remarkable Recurrent Nova M31N 2008- 12a?-Hubble Space Telescope Photometry of the 2015 Eruption
}

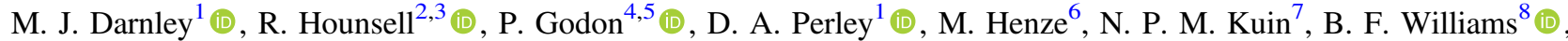 \\ S. C. Williams ${ }^{1,9}$ (1) M. F. Bode ${ }^{1}$, D. J. Harman ${ }^{1}$, K. Hornoch ${ }^{10}$, M. Link $^{11}$, J.-U. Ness ${ }^{12}$, V. A. R. M. Ribeiro ${ }^{13,14,15,16}$ (1), \\ E. M. Sion ${ }^{4}$, A. W. Shafter ${ }^{17}$ (i), and M. M. Shara ${ }^{18}$ (i) \\ ${ }^{1}$ Astrophysics Research Institute, Liverpool John Moores University, IC2 Liverpool Science Park, Liverpool, L3 5RF, UK \\ ${ }_{2}^{2}$ Department of Astronomy and Astrophysics, University of California, Santa Cruz, CA 95064, USA \\ ${ }^{3}$ Astronomy Department, University of Illinois at Urbana-Champaign, 1002 W. Green Street, Urbana, IL 61801, USA \\ ${ }^{4}$ Department of Astrophysics \& Planetary Science, Villanova University, 800 Lancaster Avenue, Villanova, PA 19085, USA \\ ${ }^{5}$ Henry A. Rowland Department of Physics \& Astronomy, Johns Hopkins University, Baltimore, MD 21218, USA \\ ${ }^{6}$ Institut de Ciències de l'Espai (CSIC-IEEC), Campus UAB, C/Can Magrans s/n, E-08193 Cerdanyola del Valles, Spain \\ ${ }^{7}$ Mullard Space Science Laboratory, University College London, Holmbury St. Mary, Dorking, Surrey RH5 6NT, UK \\ ${ }^{8}$ Department of Astronomy, Box 351580, University of Washington, Seattle, WA 98195, USA \\ ${ }^{9}$ Physics Department, Lancaster University, Lancaster, LA1 4 YB, UK \\ ${ }^{10}$ Astronomical Institute, Academy of Sciences, CZ-251 65 Ondřejov, Czech Republic \\ ${ }^{11}$ Space Telescope Science Institute, 3700 San Martin Drive, Baltimore, MD 21218, USA \\ ${ }^{12}$ XMM-Newton Observatory SOC, European Space Astronomy Centre, Camino Bajo del Castillo s/n, \\ Urb. Villafranca del Castillo, E-28692 Villanueva de la Cañada, Madrid, Spain
${ }^{13}$ CIDMA, Departamento de Física, Universidade de Aveiro, Campus de Santiago, 3810-193 Aveiro, Portugal \\ ${ }^{14}$ Instituto de Telecomunicações, Campus de Santiago, 3810-193 Aveiro, Portugal \\ ${ }^{15}$ Department of Physics and Astronomy, Botswana International University of Science \& Technology, Private Bag 16, Palapye, Botswana \\ ${ }_{16}$ Department of Astrophysics/IMAPP, Radboud University, P.O. Box 9010, 6500 GL Nijmegen, The Netherlands \\ ${ }^{17}$ Department of Astronomy, San Diego State University, San Diego, CA 92182, USA \\ ${ }^{18}$ Department of Astrophysics, American Museum of Natural History, 79th Street and Central Park West, New York, NY 10024, USA \\ Received 2017 August 7; revised 2017 September 28; accepted 2017 September 28; published 2017 November 6
}

\begin{abstract}
The recurrent nova M31N 2008-12a experiences annual eruptions, contains a near-Chandrasekhar-mass white dwarf, and has the largest mass accretion rate in any nova system. In this paper, we present Hubble Space Telescope (HST) WFC3/UVIS photometry of the late decline of the 2015 eruption. We couple these new data with archival HST observations of the quiescent system and Keck spectroscopy of the 2014 eruption. The late-time photometry reveals a rapid decline to a minimum luminosity state, before a possible recovery/rebrightening in the run up to the next eruption. Comparison with accretion disk models supports the survival of the accretion disk during the eruptions, and uncovers a quiescent disk mass accretion rate of the order of $10^{-6} M_{\odot} \mathrm{yr}^{-1}$, which may rise beyond $10^{-5} M_{\odot} \mathrm{yr}^{-1}$ during the super-soft source phase-both of which could be problematic for a number of well-established nova eruption models. Such large accretion rates, close to the Eddington limit, might be expected to be accompanied by additional mass loss from the disk through a wind and even through collimated outflows. The archival HST observations, combined with the disk modeling, provide the first constraints on the mass donor: $L_{\text {donor }}=103_{-11}^{+12} L_{\odot}, R_{\text {donor }}=14.14_{-0.47}^{+0.46} R_{\odot}$, and $T_{\text {eff,donor }}=4890 \pm 110 \mathrm{~K}$, which may be consistent with an irradiated M31 red-clump star. Such a donor would require a system orbital period $\gtrsim 5$ days. Our updated analysis predicts that the M31N 2008-12a WD could reach the Chandrasekhar mass in $<20 \mathrm{kyr}$.
\end{abstract}

Key words: accretion, accretion disks - novae, cataclysmic variables - galaxies: individual (M31) stars: individual (M31N 2008-12a) - ultraviolet: stars

\section{Introduction}

Novae are a subclass of cataclysmic variables (CVs), where a white dwarf (WD) accretes hydrogen-rich matter from a donor star within a, typically close, binary system (see Bode \& Evans 2008 and Woudt \& Ribeiro 2014 for review articles). The transferred material usually accumulates in an accretion disk around the WD, but there may also be some element of magnetic accretion at play, depending upon the strength of the WD's magnetic field. Novae are distinguishable from CVs by virtue of their typically elevated WD mass accretion rates $\left(\dot{M}_{\text {acc }}\right)$ and by the nova eruption itself-a thermonuclear runaway within the accreted envelope on the WD surface (see Starrfield et al. 1976). All novae are inherently recurrent, but their inter-eruption period depends upon the WD mass $\left(M_{\mathrm{WD}}\right)$ and $\dot{M}_{\mathrm{acc}}$. Systems that combine a large $M_{\mathrm{WD}}$ with a high $\dot{M}_{\text {acc }}$ exhibit the shortest recurrence periods and have often been observed in eruption more than once-these are the socalled recurrent novae (RNe; see Schaefer 2010). The observed recurrence periods lie in the range $1 \leqslant P_{\text {rec }} \leqslant 98$ years (see Darnley et al. 2014; Pagnotta et al. 2009, respectively), where both ends are probably limited by selection effects (see, e.g., Kato et al. 2014; Hillman et al. 2016; Shafter 2017).

In most cases, the high values of $\dot{M}_{\text {acc }}$ in RNe are driven by the elevated mass-loss rates from evolved donors (Darnley et al. 2012). This is observed to be via Roche lobe overflow of a subgiant donor (e.g., U Scorpii) or by accretion from the stellar wind of a giant (e.g., RS Ophiuchi, see e.g., Evans et al. 2008) - both mechanisms lead to an accretion disk around the WD. A handful of RNe, possibly "transient" (rather than long-term) recurrents, such as T Pyxidis, may show evidence of elevated mass transfer driven by the irradiation of their mainsequence donors (Knigge et al. 2000; Godon et al. 2014). 
Table 1

Summary of the 12 Observed Eruptions of M31N 2008-12a.

\begin{tabular}{|c|c|c|}
\hline $\begin{array}{l}\text { Eruption Date }^{\mathrm{a}} \\
\text { (UT) }\end{array}$ & $\begin{array}{c}\text { Inter-eruption } \\
\text { Timescale (days) }\end{array}$ & References \\
\hline (1992 Jan 28) & $\ldots$ & $(1),(2)$ \\
\hline (1993 Jan 03) & 341 & $(1),(2)$ \\
\hline (2001 Aug 27) & $\cdots$ & $(2),(3)$ \\
\hline 2008 Dec 25 & $\cdots$ & (4) \\
\hline 2009 Dec 02 & 342 & (5) \\
\hline 2010 Nov 19 & 352 & (2) \\
\hline 2011 Oct 22.5 & 337.5 & $(5),(6)-(8)$ \\
\hline 2012 Oct 18.7 & 362.2 & $(8)-(11)$ \\
\hline 2013 Nov $26.95 \pm 0.25$ & 403.5 & $(5),(8),(11)-(14)$ \\
\hline 2014 Oct $02.69 \pm 0.21$ & $309.8 \pm 0.7$ & $(8),(15)$ \\
\hline 2015 Aug $28.28 \pm 0.12$ & $329.6 \pm 0.3$ & (14), (16)-(18) \\
\hline 2016 Dec 12.32 & 471.72 & $(19),(20)$ \\
\hline
\end{tabular}

Notes. Compact version of a table originally published by Tang et al. (2014) and updated by Darnley et al. (2016).

${ }^{a}$ Eruption dates in parentheses have been estimated based on an extrapolation of available X-ray data (see Henze et al. 2015a).

${ }^{\mathrm{b}}$ The inter-eruption timescale is only given when consecutive eruptions were detected (assuming $P_{\text {rec }} \simeq 1$ year).

References. (1) White et al. (1995), (2) Henze et al. (2015a), (3) Williams et al. (2004), (4) Nishiyama \& Kabashima (2008), (5) Tang et al. (2014), (6) Korotkiy \& Elenin (2011), (7) Barsukova et al. (2011), (8) Darnley et al. (2015c), (9) Nishiyama \& Kabashima (2012), (10) Shafter et al. (2012), (11) Henze et al. (2014), (12) Tang et al. (2013), (13) Darnley et al. (2014), (14) Darnley et al. (2016), (15) Henze et al. (2015b), (16) Darnley et al. (2015a), (17) Darnley et al. (2015b), (18) Henze et al. (2015c), (19) Itagaki et al. (2016), (20) Henze et al. (2017).

M31N 2008-12a, an RN residing within M31, is the most extreme nova system discovered to date. With an observed $P_{\text {rec }} \simeq 1$ year, it is the prototype of a newly emerging class of "rapidly recurring novae"- those with $P_{\text {rec }} \lesssim 10$ years. First detected in 2008, M31N 2008-12a has been discovered in eruption every year since (2008-2016; Darnley et al. 2014, 2015c, 2016; Itagaki et al. 2016), with three previous eruptions recovered from archival X-ray observations (1992, 1993, 2001; Henze et al. 2014; Tang et al. 2014). For reference, the observed eruption history is summarized in Table 1 (see Darnley et al. 2016 for a detailed description). By analyzing the eight eruptions between 2008 and 2015, Darnley et al. (2016, hereafter DHB16) reported $P_{\text {rec }}=347 \pm 10$ days. However, when including the earlier X-ray detections, Henze et al. (2015a, hereafter HDK15) suggested that $P_{\text {rec }}$ could even be as short as $174 \pm 10$ days.

These rapid-fire eruptions of $\mathrm{M} 31 \mathrm{~N} 2008-12 \mathrm{a}$ are powered by the most massive accreting WD yet discovered. Studies of the 2013 eruption yielded $M_{\mathrm{WD}}>1.3 M_{\odot}$ (Tang et al. 2014, hereafter TBW14), with a more recent determination of $M_{\mathrm{WD}}=1.38 M_{\odot}($ Kato et al. 2015). We note that the WD mass was not measured directly, only estimated based on modeling of the system. Those same models required very large accretion rates, $\dot{M}_{\text {acc }}>1.7 \times 10^{-7} M_{\odot} \mathrm{yr}^{-1}$ and $\dot{M}_{\text {acc }}=$ $1.6 \times 10^{-7} M_{\odot} \mathrm{yr}^{-1}$, respectively. Under the assumption of spherical ejecta, Henze et al. (2015b, hereafter HND15) concluded that the quantity of ejected hydrogen was $M_{\mathrm{e}, \mathrm{H}}=(2.6 \pm 0.4) \times 10^{-8} M_{\odot}$, broadly consistent with the total ejected mass prediction of $M_{\mathrm{e}}=6 \times 10^{-8} M_{\odot}$ from Kato et al. (2015). This indicates a mass accretion efficiency of $\sim 63 \%$; hence, not only is the WD massive, but it is also growing.

Darnley et al. (2014, hereafter DWB14) and TBW14 both illustrated the rapid optical development of the 2013 eruption; Henze et al. (2014, hereafter HND14) and TBW14 noted the rapid X-ray development. DHB16 combined all data from the nearly identical 2013, 2014, and 2015 eruptions to determine that the optical decay time $\left(t_{2}\right.$; the time to decay two magnitudes from the peak luminosity) is only $1.65 \pm$ 0.04 days, and $t_{3}=2.47 \pm 0.06$ days. The accompanying super-soft X-ray source (SSS) "turned on" only $5.6 \pm 0.7$ days after the 2015 eruption and turned off after $18.6 \pm 0.7$ days (DHB16); only the Galactic RN V745 Scorpii displays more rapid X-ray evolution (Page et al. 2015).

DHB16 also presented a detailed analysis of the combined spectra of the 2012-2015 eruptions. The earliest post-eruption spectra show fleeting evidence of very high-velocity $\left(v_{\mathrm{ej}} \simeq\right.$ $13,000 \mathrm{~km} \mathrm{~s}^{-1}$ ) outflows. DHB16 proposed that these could be due to a high level of ejecta collimation in the polar direction, almost along the line of sight. Darnley et al. (2017, hereafter DHG17) reported similar high-velocity material surrounding the far-UV N V (1240 ̊) emission line three days after the 2015 eruption; again, this was linked to possible ejecta collimation or jets from the eruption.

Hints of ejecta deceleration were first reported by TBW14. The DHB16 analysis of the combined 2012-2015 spectroscopy found clear evidence of significant ejecta deceleration, consistent with the adiabatic expansion of a forward shock (cf. Bode \& Kahn 1985). DHS15 and DHB16 both proposed that this deceleration could be caused by the ejecta interacting with pre-existing circumbinary material. Given that the circumbinary regime should be cleared by each annual eruption, this environment must be regularly resupplied. Therefore, DHB16 proposed that the M31N 2008-12a donor should be a giant with a significant stellar wind, and not Roche lobe overflow.

Utilizing the Swift observatory, Kato et al. (2016) undertook the first targeted survey to detect the long-predicted X-ray flash precursor to a nova eruption (see, e.g., Starrfield et al. 1990; Krautter 2002). The campaign was unsuccessful, possibly because of the earlier than predicted 2015 eruption, or because the flash was absorbed by pre-existing material surrounding the system. At the time, there was no strong evidence constraining the mass donor in the system, therefore Kato et al. (2016) favored the former explanation.

Containing a growing WD that is already close to the Chandrasekhar limit, M31N 2008-12a is therefore the leading pre-explosion supernova Type Ia candidate system. Hubble Space Telescope (HST) spectroscopy of the 2015 eruption conducted by DHG17 found no evidence of neon within the ejecta. However, as discussed by those authors, that single result still cannot completely rule out the presence of an $\mathrm{ONe}$ WD in the system. Either way, DHG17 argued that the lack of an observational signature of $\mathrm{Ne}$ may in itself indicate that the M31N 2008-12a WD is growing in mass. That is, either a CO WD has grown to the Chandrasekhar limit, or a large enough He layer has been accumulated to shield an underlying $\mathrm{ONe}$ WD from the nova eruptions.

In this paper, we present the results of an HST program to study the late decline of the predicted 2015 eruption of M31N 2008-12a and an updated analysis of archival HST and Keck observations of the system. In Section 2, we describe our 
Table 2

Log of Observations of the Eruptions of M31N 2008-12a Referred to in This Paper

\begin{tabular}{|c|c|c|c|c|c|c|c|c|}
\hline Eruption & Facility & Instrument & $\begin{array}{l}H S T \\
\text { Visit }\end{array}$ & $\begin{array}{c}\text { Date } \\
\text { (midpoint) }\end{array}$ & $\begin{array}{c}\text { Start } \\
t-t_{0}\end{array}$ & $\begin{array}{l}\text { End } \\
\text { (days) }\end{array}$ & Orbits & $\begin{array}{l}\text { Exposure } \\
\text { Time (ks) }\end{array}$ \\
\hline 2014 & Keck I & LRIS & $\cdots$ & 2014 Oct 21.50 & 18.80 & 18.82 & $\cdots$ & 1.2 \\
\hline 2015 & $H S T$ & WFC3/UVIS & 4 & 2015 Sep 10.64 & 13.27 & 13.44 & 3 & 6.8 \\
\hline 2015 & $H S T$ & WFC3/UVIS & 5 & 2015 Sep 17.66 & 20.29 & 20.47 & 3 & 6.8 \\
\hline 2015 & $H S T$ & WFC3/UVIS & 6 & 2015 Sep 23.62 & 26.26 & 26.43 & 3 & 6.8 \\
\hline 2015 & $H S T$ & WFC3/UVIS & 7 & 2015 Sep 30.58 & 33.22 & 33.39 & 3 & 6.8 \\
\hline
\end{tabular}

observations; in Section 3, we present the photometric data. In Section 4, we explore models of the accretion disk in M31N 2008-12a. Finally, in Sections 5 and 6, we discuss our findings and present our subsequent conclusions.

While this manuscript was being prepared, the 2016 eruption of M31N 2008-12a was detected by Itagaki et al. (2016). The observations of the 2016 eruption will be presented in Henze et al. (2017).

\section{Observations}

\subsection{Hubble Space Telescope Observations}

Twenty orbits of HST Cycle 23 time were awarded to collect early-time UV spectroscopic observations (eight orbits) and late-time imaging of the 2015 eruption of M31N 2008-12a (proposal ID: 14125). The results of the spectroscopy are presented in DHG17. The 2015 eruption was discovered on 2015 August 28.425 UT by an automated monitoring program on the Las Cumbres Observatory $2 \mathrm{~m}$ telescope ${ }^{19}$ on Hawai' $\mathrm{i}$ (Darnley et al. 2015a; see DHB16 for full details). The HST photometric observations were conducted between 2015 September 10 and September 30; a log of these observations is provided in Table 2.

We employed 12 HST orbits, split into four visits, to collect photometry of M31N 2008-12a using Wide Field Camera 3 (WFC3) in UVIS mode. Each visit used identical observing strategies and were approximately one week apart, starting at $\Delta t \simeq 14$ days (post-eruption). Observations were obtained using the WFC3/UVIS F225W, F275W, F336W, F475W, and F814W filters.

For each filter, a two-point dither was applied to enable the removal of detector defects. To reduce readout overheads, WFC3/UVIS was operated in a $2 \mathrm{k} \times 2 \mathrm{k}$ windowed mode utilizing the UVIS2-2K2C-SUB aperture. This part of the chip was selected for its superior performance against charge transfer efficiency (CTE) loss; to further mitigate such effects, we included a "post-flash" signal of 9-12 electrons.

The WFC3/UVIS data were reduced using the STScI calwf3 pipeline (v3.1.6; see Dressel 2012), with CTE correction manually applied via the wfc3uv_ctereverse_parallel code (v2015.07.22 $2^{20}$; see also Anderson et al. 2012). Photometry of the WFC3/UVIS data was then performed on individual exposures using DOLPHOT (v2.0 ${ }^{21}$; Dolphin 2000; following the standard procedure and parameters for WFC3/UVIS given in the manual). For comparative purposes, photometry was also carried out using the combined exposures per epoch for each filter. All data were aligned, and

\footnotetext{
19 Formerly known as the Faulkes Telescope North.

${ }^{20}$ http://www.stsci.edu/hst/wfc3/tools/cte_tools

21 http://americano.dolphinsim.com/dolphot
}

the final combined images were created using the Drizzlepac (v2.0.2) astrodrizzle package. Photometry was obtained via the PyRAF phot package (v2.2). The results from the DOLPHOT and phot methods are consistent. For comparison with previous work, we adopt the DOLPHOT photometry, which is presented in Table 3.

\subsection{Keck Spectroscopy of the 2014 Eruption}

DHB16 observed that photometrically, the 2013, 2014, and 2015 eruptions were essentially identical; the same is true of the spectra from the 2012-2015 eruptions. Therefore, to support the late-time HST photometry of the 2015 eruption, we also utilize a Keck spectrum of the 2014 eruption taken 18.81 days after that eruption.

This 2014 Keck spectrum has not been published until now. It was collected using the Low Resolution Imaging Spectrometer (LRIS; Oke et al. 1995; McCarthy et al. 1998; Rockosi et al. 2010), which is mounted at the Cassegrain focus of the Keck I telescope on Maunakea, Hawai'i. The spectrum was obtained through the standard low-resolution configuration using the 400/3000 grism (blue camera) and 400/8500 grating (red camera), providing continuous coverage from the atmospheric cutoff to approximately $10300 \AA$. However, as the nova had faded significantly, crowding and confusion with nearby stars in M31 had started to be problematic; therefore, the object is only clearly detected in the blue camera. Only data with $\lambda<5600 \AA$ are analyzed here.

\subsection{Archival Quiescent Data}

The Panchromatic Hubble Andromeda Treasury (PHAT; Dalcanton et al. 2012) was a broadband, multicolor, NUV-NIR HST survey of the bulge and northeastern disk of M31. As part of the PHAT survey, M31N 2008-12a was observed between eruptions a number of times with HST. Initial results from analysis of these data were published in DWB14 and TBW14. Both of those works analyzed the optical and NUV HST data, finding evidence for a very blue source coincident with M31N 2008-12a, indicating the presence of a luminous accretion disk. Although the available HST NIR data were also analyzed, DWB14 and TBW14 only presented upper limits on the quiescent photometry of M31N 2008-12a, which was severely blended with nearby sources in the NIR. These upper limits did not place firm constraints on the nature of the donor, only excluding the most luminous red giants (such as that found in the TCoronae Borealis system). Notably, the initial analysis of the quiescent SED indicated an accretion disk roughly similar in flux distribution, albeit brighter, to that in the RS Oph system; a donor of similar luminosity to the red giant in RS Oph was not ruled out by DWB14. 
Table 3

Hubble Space Telescope WFC3/UVIS NUV and Visible Photometry of M31N 2008-12a Following the Late Decline of the 2015 Eruption

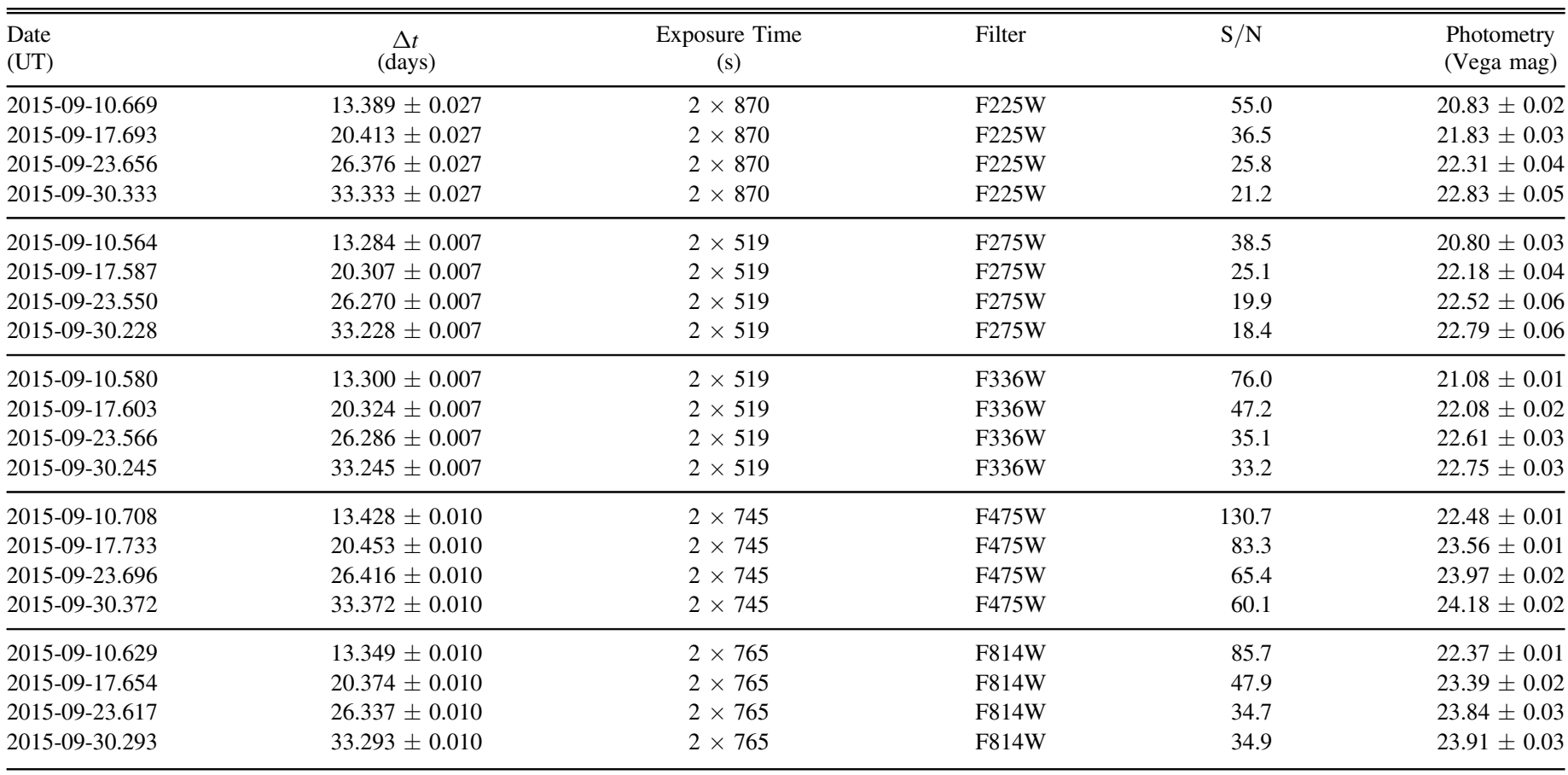

Williams et al. (2014a) released the NUV to NIR photometric catalog from the PHAT survey, which included the quiescent photometry of M31N 2008-12a. These photometry are provided in Table 4 and are consistent with the independent analysis by DWB14 and TBW14. However, the analysis undertaken by Williams et al. (2014a) was able to successfully de-blend the sources around M31N 2008-12a in the NIR, yielding F110W and F160W photometry of the quiescent system. This superior NIR deblending was achieved by simultaneous fitting of the higher spatial resolution $\mathrm{F} 475 \mathrm{~W}$ data with the NIR data. These F475W data have spatial resolution better by more than a factor of two and allowed for a much more robust deblending of crowded sources.

The observations reported in Williams et al. (2014a) are from the first set of PHAT visits and are computed over two separate $H S T$ visits. Data from another pair of visits are also available, and the PHAT collaboration have generously supplied their photometry of M31N 2008-12a from each of the four HST visits; these data are also shown in Table 4. The quiescent photometry reported by DWB14 and TBW14 are combined from observations at different phases in the full eruption cycle of M31N 2008-12a and from different eruption cycles (as noted by both of those papers).

\section{Light Curve Analysis}

The five-band HST photometry was presented in Table 3, and the subsequent light curves are presented in Figure 1. In the subfigures, the four epochs of the HST WFC3/UVIS observations (black data points) are compared with the template M31N 2008-12a eruption light curves from other telescopes. These eruption templates are constructed from Bézier smoothed light curves of the almost identical 2013-2015 eruptions (DWB14, DHS15, DHB16). The uncertainties on the smoothed light curves are computed based on the method employed by Ashall et al. (2016). Here, we compare to the closest filter in wavelength to the $H S T$ filters. The F275W filter is compared to the Swift UVW1 data (central wavelength $2600 \AA$ ), F336W to the Sloan $u^{\prime}$-band (the Sloan ground-based data are converted from the AB system to the Vega system in this plot), F475W to $B$, and F814W to $i^{\prime}$; the Swift UVM2 filter (2250 $\AA$ ) is used for comparison to the $\mathrm{F} 225 \mathrm{~W}$ data, but these data are not particularly extensive. The solid vertical lines in each plot indicate the epochs of the SSS turn on and turn off. The horizontal lines indicate the minimum photometry from the two visits of the PHAT survey (see Table 4).

The HST WFC3/UVIS F225W data are well-fit by a power law of the form $f \propto t^{\alpha}$, where $\alpha=-2.04 \pm 0.16\left(\chi_{\text {/dof }}^{2}=\right.$ 2.2). We note that this decline is therefore consistent with the "middle" relation predicted by the universal decline law of Hachisu \& Kato $(2006,2007 ; \alpha=-1.75)$. This may be connected to the lack of strong emission lines seen in the equivalent region of the NUV spectrum (see DHG17). We also note that DHB16 found that a power-law fit to the $u^{\prime}$-band decline of the 2015 eruption, between days 8 and 20, was consistent with the predicted "middle" decline law. The HST data from the other filters all show significant deviation from a single power law, when taken in isolation and when compared to the eruption template data-all these filters contain strong flux contributions from lines.

\subsection{Quiescent Data}

Comparison between the HST imaging of the 2015 eruption and the archival data confirm that the object proposed by DWB14 and TBW14 as a quiescent system is associated with the eruptions of M31N 2008-12a. In Table 4, we also indicated the epoch of the archival PHAT HST visits with respect to the M31N 2008-12a eruption cycle. The closest PHAT observations to a known eruption are those from 2011 January, which took place 67 days after the 2010 November eruption, which is significantly later, post-eruption, than the late-time decline data 
Table 4

PHAT Multicolor NUV, Optical, and NIR Photometry of M31N 2008-12a, in Part from Williams et al. (2014a)

\begin{tabular}{|c|c|c|c|c|c|c|c|c|}
\hline \multirow{2}{*}{$\begin{array}{l}\text { Date } \\
\text { (UT) }\end{array}$} & \multicolumn{2}{|c|}{ Observed Eruptions } & \multicolumn{2}{|c|}{ Predicted Eruptions } & \multirow{2}{*}{$\begin{array}{c}H S T \\
\text { Instrument }\end{array}$} & \multirow{2}{*}{ Filter } & \multirow{2}{*}{$\begin{array}{l}\text { Exposure } \\
\text { Time } \\
\text { (s) }\end{array}$} & \multirow{2}{*}{ Photometry } \\
\hline & $\begin{array}{l}\Delta t_{\mathrm{after}} \\
\text { (days) }\end{array}$ & $\begin{array}{c}\Delta t_{\text {before }} \\
\text { (days) }\end{array}$ & $\begin{array}{l}\Delta t_{\mathrm{after}} \\
\text { (days) }\end{array}$ & $\begin{array}{l}\Delta t_{\text {before }} \\
(\text { days })\end{array}$ & & & & \\
\hline 2011 Jan 25.21 & 67 & 270 & 67 & $115 \pm 26$ & WFC3/UVIS & F275W & 350 & $23.13 \pm 0.12^{\mathrm{a}}$ \\
\hline 2011 Jan 25.23 & 67 & 270 & 67 & $115 \pm 26$ & WFC3/UVIS & F275W & 660 & $22.98 \pm 0.07^{\mathrm{a}}$ \\
\hline 2011 Aug 31.51 & 285 & 52 & $103 \pm 26$ & 52 & WFC3/UVIS & F275W & 350 & $22.73 \pm 0.09$ \\
\hline 2011 Aug 31.53 & 285 & 52 & $103 \pm 26$ & 52 & WFC3/UVIS & F275W & 575 & $22.53 \pm 0.06$ \\
\hline 2011 Jan 25.20 & 67 & 270 & 67 & $115 \pm 26$ & WFC3/UVIS & F336W & 550 & $23.07 \pm 0.05^{\mathrm{a}}$ \\
\hline 2011 Jan 25.22 & 67 & 270 & 67 & $115 \pm 26$ & WFC3/UVIS & F336W & 800 & $23.01 \pm 0.04^{\mathrm{a}}$ \\
\hline 2011 Aug 31.51 & 285 & 52 & $103 \pm 26$ & 52 & WFC3/UVIS & F336W & 550 & $22.59 \pm 0.04$ \\
\hline 2011 Aug 31.52 & 285 & 52 & $103 \pm 26$ & 52 & WFC3/UVIS & F336W & 700 & $22.59 \pm 0.04$ \\
\hline 2010 Aug 07.53 & 248 & 104 & $68 \pm 26$ & 104 & $\mathrm{ACS} / \mathrm{WFC}$ & F475W & 600 & $24.08 \pm 0.02^{\mathrm{a}}$ \\
\hline 2010 Aug 07.53 & 248 & 104 & $68 \pm 26$ & 104 & ACS/WFC & F475W & 370 & $24.06 \pm 0.03^{\mathrm{a}}$ \\
\hline 2010 Aug 07.54 & 248 & 104 & $68 \pm 26$ & 104 & $\mathrm{ACS} / \mathrm{WFC}$ & F475W & 370 & $24.01 \pm 0.03^{\mathrm{a}}$ \\
\hline 2010 Aug 07.54 & 248 & 104 & $68 \pm 26$ & 104 & ACS/WFC & F475W & 370 & $24.08 \pm 0.03^{\mathrm{a}}$ \\
\hline 2012 Jan 10.12 & 80 & 282 & 80 & $118 \pm 26$ & $\mathrm{ACS} / \mathrm{WFC}$ & F475W & 700 & $24.46 \pm 0.03$ \\
\hline 2012 Jan 10.13 & 80 & 282 & 80 & $118 \pm 26$ & ACS/WFC & $\mathrm{F} 475 \mathrm{~W}$ & 360 & $24.48 \pm 0.04$ \\
\hline 2012 Jan 10.13 & 80 & 282 & 80 & $118 \pm 26$ & $\mathrm{ACS} / \mathrm{WFC}$ & F475W & 360 & $24.43 \pm 0.04$ \\
\hline 2012 Jan 10.14 & 80 & 282 & 80 & $118 \pm 26$ & ACS/WFC & $\mathrm{F} 475 \mathrm{~W}$ & 470 & $24.51 \pm 0.03$ \\
\hline 2010 Aug 07.45 & 248 & 104 & $68 \pm 26$ & 104 & ACS/WFC & F814W & 350 & $23.87 \pm 0.05^{\mathrm{a}}$ \\
\hline 2010 Aug 07.46 & 248 & 104 & $68 \pm 26$ & 104 & $\mathrm{ACS} / \mathrm{WFC}$ & F814W & 700 & $23.80 \pm 0.03^{\mathrm{a}}$ \\
\hline 2010 Aug 07.47 & 248 & 104 & $68 \pm 26$ & 104 & $\mathrm{ACS} / \mathrm{WFC}$ & F814W & 455 & $23.83 \pm 0.04^{\mathrm{a}}$ \\
\hline 2012 Jan 10.02 & 80 & 282 & 80 & $118 \pm 26$ & ACS/WFC & F814W & 350 & $23.98 \pm 0.05$ \\
\hline 2012 Jan 10.05 & 80 & 282 & 80 & $118 \pm 26$ & ACS/WFC & F814W & 800 & $23.97 \pm 0.04$ \\
\hline 2012 Jan 10.06 & 80 & 282 & 80 & $118 \pm 26$ & ACS/WFC & F814W & 550 & $23.99 \pm 0.04$ \\
\hline 2011 Jan 25.27 & 67 & 270 & 67 & $115 \pm 26$ & WFC3/IR & F110W & 800 & $24.19 \pm 0.05^{\mathrm{a}}$ \\
\hline 2011 Aug 31.58 & 285 & 52 & $103 \pm 26$ & 52 & WFC3/IR & F110W & 700 & $23.71 \pm 0.03$ \\
\hline 2011 Jan 25.26 & 67 & 270 & 67 & $115 \pm 26$ & WFC3/IR & F160W & 400 & $24.1 \pm 0.2^{\mathrm{a}}$ \\
\hline 2011 Jan 25.28 & 67 & 270 & 67 & $115 \pm 26$ & WFC3/IR & F160W & 400 & $24.0 \pm 0.2^{\mathrm{a}}$ \\
\hline 2011 Jan 25.29 & 67 & 270 & 67 & $115 \pm 26$ & WFC3/IR & F160W & 400 & $23.9 \pm 0.2^{\mathrm{a}}$ \\
\hline 2011 Jan 25.29 & 67 & 270 & 67 & $115 \pm 26$ & WFC3/IR & F160W & 500 & $24.2 \pm 0.2^{\mathrm{a}}$ \\
\hline 2011 Aug 31.57 & 285 & 52 & $103 \pm 26$ & 52 & WFC3/IR & F160W & 400 & $23.5 \pm 0.1$ \\
\hline 2011 Aug 31.59 & 285 & 52 & $103 \pm 26$ & 52 & WFC3/IR & F160W & 400 & $23.5 \pm 0.1$ \\
\hline 2011 Aug 31.59 & 285 & 52 & $103 \pm 26$ & 52 & WFC3/IR & F160W & 400 & $23.4 \pm 0.1$ \\
\hline 2011 Aug 31.60 & 285 & 52 & $103 \pm 26$ & 52 & WFC3/IR & F160W & 400 & $23.3 \pm 0.1$ \\
\hline
\end{tabular}

Note.

${ }^{a}$ Data derived directly from Williams et al. (2014a); the remainder have been provided directly by the PHAT collaboration.

collected for this paper. We also note that the 2011 August observations took place 52 days before the 2011 October eruption.

HDK15 presented evidence that M31N 2008-12a may erupt every $\sim 6$ months, rather than annually. If this is the case, we must also assess whether the interpretation of the archival HST data may be affected by unobserved eruptions. The typical eruption date uncertainty is 26 days (HDK15). If we utilize the dates of the observed eruptions but assume a $\sim 6$ month cycle (see HDK15), we can investigate how close to an unobserved eruption each PHAT visit potentially occurred (recorded in Table 4). The only observations of note here are those from 2010 August, which may lie $68 \pm 26$ days after an unobserved early 2010 eruption. The F475W and F814W data from that time are significantly brighter than those from 2012 January (80 days after the 2011 October eruption), which suggests that these data may be coincident with the late decline of an early (but missed) 2010 eruption.
By assuming that all M31N 2008-12a eruptions are essentially identical, we can roughly fit the 2010 August HST observations to the 2015 eruption late-decline observations. Therefore, we would predict that a missed eruption of M31N 2008-12a could have occurred on 2010 July $09_{-3}^{+4}$ (see the light blue data points in Figure 1). However, data from PTF rule out an additional eruption between 2010 June 30 and the date of the observed 2010 November eruption (Cao et al. 2012; M. M. Kasliwal 2017, private communication). As such, we conclude that all PHAT data of M31N 2008-12a were taken at least 67 days after an eruption, and that they represent observations of the inter-eruption, or quiescent, period. We stress that this does not rule out the possibility of an early 2010 eruption occurring before this window.

\subsection{A "Folded" Eruption Cycle}

The HST data covering the quiescent system are admittedly sparse and spread across multiple eruption cycles. However, 


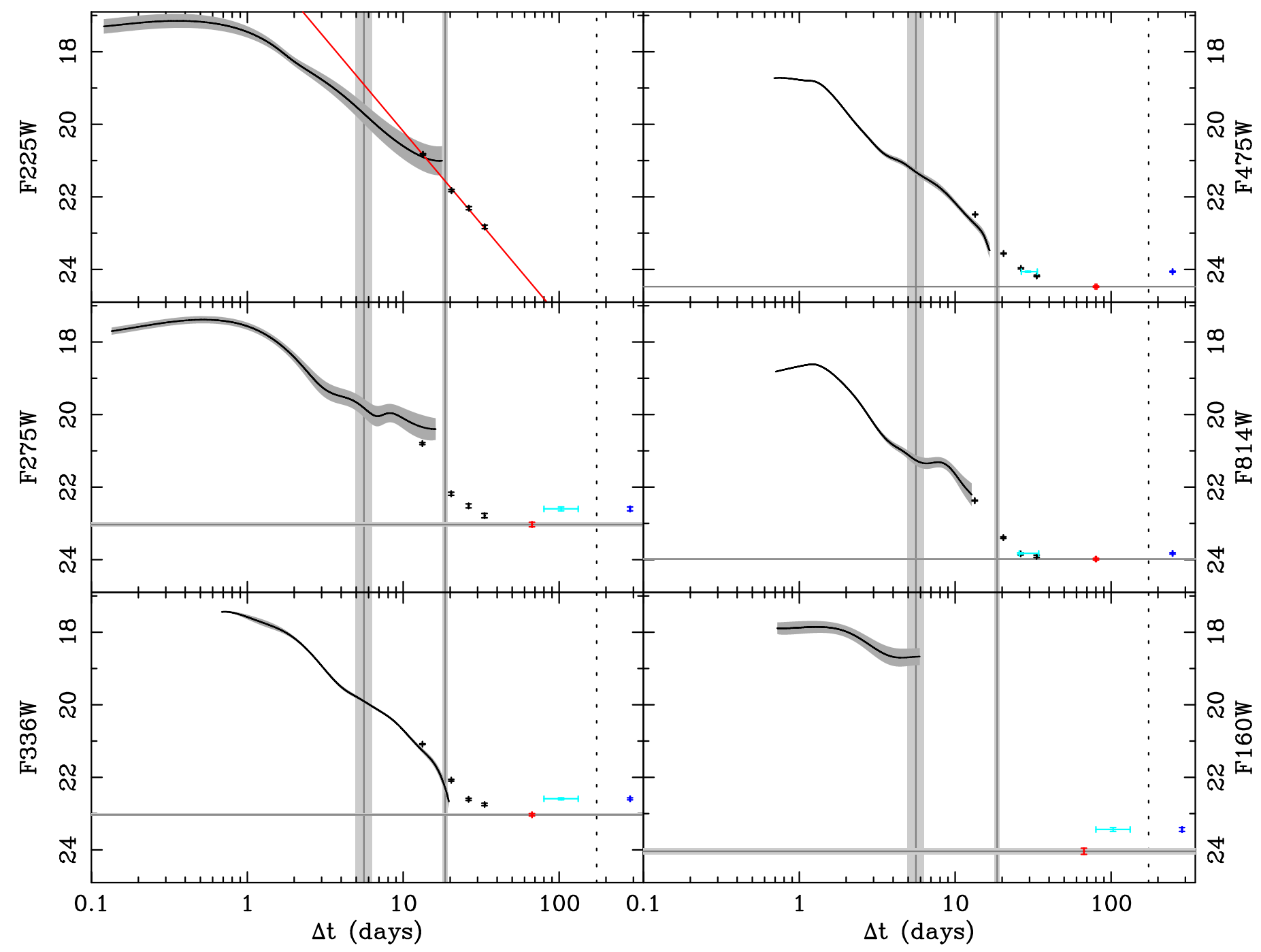

Figure 1. Near-ultraviolet through optical HST WFC3/UVIS photometry of the 2015 eruption of M31N 2008-12a (black data points). The time axes runs from 0.1 days post-eruption up to 347 days - the mean observed recurrence period. The vertical dashed line indicates the proposed 147 day recurrence period (HDK15). The horizontal lines, where shown, indicate the faintest detection of the two PHAT epochs-assumed to be the quiescence level. The vertical gray lines indicate the turn on and turn off times of the SSS from the 2015 eruption (the shaded areas show their associated uncertainties). The solid black lines show combined and smoothed (Bézier curve) photometry from the 2013, 2014, and 2015 eruptions of M31N 2008-12a, with the surrounding shaded area indicating the $1 \sigma$ uncertainty; these smoothed lines are provided for illustrative and contextual purposes only-the HST and ground-based/Swift data are taken through similar, but different, filters. The solid red line in F225W shows the best-fit power law of index $-2.04 \pm 0.16$. The red data points indicate the faintest archival PHAT photometry following a detected eruption, the dark blue points indicate additional PHAT photometry (assuming a year-long cycle), and the light blue points are the same PHAT photometry points extrapolated to a predicted missed eruption (based on a six-month recurrence period).

under the assumption of essentially identical eruptions (Schaefer 2010, DHB16), M31N 2008-12a appears to take $\sim 70$ days to return to quiescence, i.e., to reach a minimum flux following an eruption. From this point, the luminosity of the system appears to increase in the lead up to the next eruption, consistent with the findings of M. Henze et al. (2017, in preparation).

The RN RS Oph is perhaps the best-studied Galactic nova both during eruption and at quiescence (see Evans et al. 2008 and references therein). Following the 2006 eruption of RS Oph, the system was observed to decline to an optical minimum before the flux began to systematically increase. The increase in flux was more prominent in bluer bands (Darnley et al. 2008) and coincided with the resumption of optical flickering (Worters et al. 2007). These observations were proposed to indicate the reestablishment of accretion post-eruption, following the destruction or severe disruption of that disk.
By mapping the quiescent PHAT data onto the template light curves, we can combine these multicolor data into two distinct quiescent epochs, based on their approximate phase in the eruption cycle. The first (red points in Figure 1), $\sim 75$ days post-eruption, represents the approximate minimum luminosity state; the second (dark blue points), $\sim 270$ days post-eruption, shows a state of increased flux. We again note that the lighter blue data points in Figure 1 indicate one possible realization of a six-month recurrence period, a realization that is ruled out by PTF data (see Section 3.1).

\subsection{Spectral Energy Distribution}

Optical and NUV photometric observations of the 2014 eruption of $\mathrm{M} 31 \mathrm{~N} 2008-12 \mathrm{a}$ indicated that, due to the low ejected mass, the unusually low maximum radius of the expanding pseudo-photosphere resulted in emission peaking in 

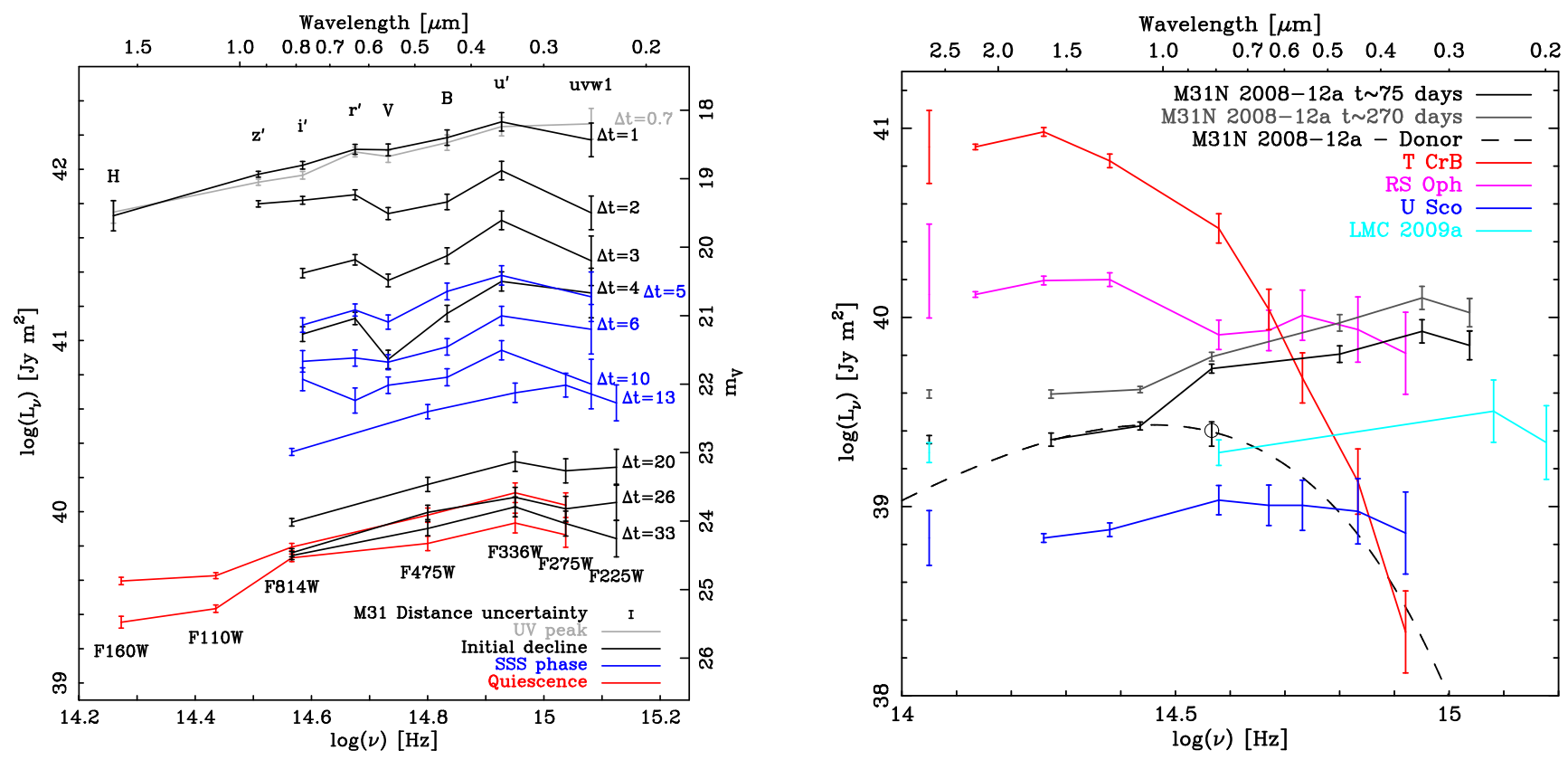

Figure 2. Distance- and extinction- ( $E_{B-V}=0.1$; DHG17) corrected SEDs showing (left) the evolving SED of the 2015 eruption (blue points indicate epochs when the SSS emission was visible, red points indicate the archival photometry) and (right) the quiescent M31N 2008-12a compared to the quiescent RNe RS Oph, T CrB, U Sco, and LMC 2009a. The black data show M31N 2008-12a at minimum ( 75 days post-eruption), and the gray data show it at an elevated state $(t \simeq 270$ days). Throughout, units have been chosen to allow comparison with similar plots in Schaefer et al. (2010, see their Figure 71 ) and DWB14 (see their Figure 4). The central wavelength locations of the Johnson-Cousins, Sloan, HST, and Swift filters are shown to assist the reader; see the keys for line identifications. For each system, the photometric uncertainties are relatively small, the indicated error bars are dominated by extinction uncertainties, and the isolated error bar to the left of each SED indicates the systematic distance uncertainty. The single circled black data point indicates the excess donor flux once the accretion disk model (see Section 4.5) has been subtracted. The dashed black line indicates a black body fit to the M31N 2008-12a donor SED (see Section 5.2).

the UV (DHS15). For all other well-observed novae, this peak occurs at visible wavelengths.

DHB16 presented a more comprehensive series of SEDs following the NIR (H-band) through NUV (Swift uvw1) decline of the 2015 eruption of M31N 2008-12a spanning $t \sim 1-10$ days post-eruption. In Figure 2, we reproduce the SED evolution plot from DHB16 and include the HST WFC3/ UVIS photometry from the 2015 eruption of M31N 2008-12a $(t \sim 13,20,26$, and 33 days post-eruption). We also include the updated quiescent photometry from archival HST observations. In Section 4, we will use these new data in conjunction with model accretion disks to constrain the mass accretion rates. The nature of the quiescent system is explored in Section 5.2.

\section{Modeling the Accretion Disk}

In this section, and subsequently in Section 5, we discuss in detail our models and interpretation of the accretion disk in M31N 2008-12a. Here, for clarity, we formally define some of the accretion rate terminology that we employ.

The models, discussed below, generate the disk mass accretion rate $(\dot{M})$, whereas the existing M31N 2008-12a eruption models of Kato et al. (2014, 2015, 2016, 2017a, $2017 b)$ are concerned with the WD mass accretion rate $\left(\dot{M}_{\text {acc }}\right)$, the amount of material that falls onto the WD surface itself.

In this work, we will also consider mass loss from the disk via a disk wind $\left(\dot{M}_{\text {wind }}\right)$ and mass loss from any outflows from the WD or the disk-WD boundary layer $\left(\dot{M}_{\mathrm{bl}}\right)$ such that

$$
\dot{M}_{\text {acc }}=\dot{M}-\dot{M}_{\text {wind }}-\dot{M}_{\mathrm{bl}} \text {. }
$$

For most novae, $\dot{M}$ is low; therefore, it is expected that $\dot{M}_{\text {wind }}-\dot{M}_{\mathrm{bl}}$ are small, and as such $\dot{M}_{\text {acc }} \simeq \dot{M}$.

\subsection{Disk Models}

The tlusty, synspec, rotin, and disksyn suite of codes (Hubeny 1988; Hubeny et al. 1994; Hubeny \& Lanz 1995) are employed to generate synthetic spectra of stellar atmospheres and disks. These include the treatment of hydrogen quasi-molecular satellite lines (low temperature) and NLTE approximation (high temperature). synspec generates continuum spectra with absorption lines. In the present work, we do not generate emission lines (see, e.g., Puebla et al. 2007 for a physical description of emission-line profiles from disks in CVs). For disk spectra, we assume solar abundances, and for stellar spectra, we vary the abundances as required.

The tlusty code is first run to generate one-dimensional (vertical) stellar atmosphere structures for a given surface gravity, effective temperature, and surface composition of the star. $\mathrm{H}$ and $\mathrm{He}$ are treated explicitly, whereas $\mathrm{C}, \mathrm{N}$, and $\mathrm{O}$ are treated implicitly (Hubeny \& Lanz 1995).

The synspec code takes the tlusty stellar atmosphere model as an input and generates a synthetic stellar spectrum over a given wavelength range from below $900 \AA$ and into the optical. The synspec code then derives the detailed radiation and flux distribution of the continuum and lines to generate the output spectrum (Hubeny \& Lanz 1995). synspec has its own chemical abundances input to generate lines for the chosen species. For temperatures $>35,000 \mathrm{~K}$, the approximate NLTE line treatment is turned on in synspec.

Rotational and instrumental broadening, as well as limb darkening (see Wade \& Hubeny 1998), are then reproduced using the rotin routine. In this manner, we generated WD synthetic spectra covering a wide range of temperatures and gravities, all with solar composition. 
The disk spectra are generated by dividing the disk into annuli, with radius $r_{i}$ and effective surface temperature $T\left(r_{i}\right)$ obtained from the standard disk model for a given WD mass $M_{\mathrm{WD}}$ and mass-loss rate.

Utilizing the input parameters of the disk mass accretion rate $(\dot{M}), M_{\mathrm{WD}}$, the radius of the WD $R_{\mathrm{WD}}$, the inner radius of the disk $R_{0}$, and the outer radius of the disk $R_{\text {disk, tlusty }}$ generates a one-dimensional vertical structure for each disk annulus (Wade \& Hubeny 1998).

In the standard disk model, the radius $R_{0}$ is the boundary at which the "no shear" condition is imposed: $d \Omega / d R=0$ (Pringle 1977). Consequently, the assumed value of $R_{0}$ affects the entire solution (not just the boundary) and the temperature profile of the disk.

For moderate disk mass accretion rates, $\dot{M} \sim 10^{-8} M_{\odot} \mathrm{yr}^{-1}$, the boundary layer between the disk and the WD, that region where the angular velocity in the disk decreases from its Keplerian value $\Omega_{K}$ to match the more slowly rotating WD surface $\Omega_{\star}$, is very small $\left(\sim 0.01 R_{\mathrm{WD}}\right)$ and one can therefore assume $R_{0}=R_{\mathrm{WD}}$ (Pringle 1977).

In our present modeling, $R_{0}$ is allowed to be larger than the radius of the $\mathrm{WD}, R_{0}>R_{\mathrm{WD}}$, to accommodate a larger boundary layer (see Godon et al. 2017 for a description of this modified disk model). As $\dot{M}$ increases, the boundary layer becomes larger (Popham \& Narayan 1995). As $\dot{M}$ reaches the Eddington accretion limit, the size of the boundary layer rises to the order of the radius of the WD ( $R_{0} \sim R_{\mathrm{WD}}$; Godon 1997).

Given the large quiescent luminosity and high ejection velocities, DHB16 and DHG17 proposed that the system inclination must be low. Although high-inclination systems are not formally ruled out, we note that the large observed disk luminosity would require a significant increase in any derived $\dot{M}$ as the assumed inclination increases.

To model the M31N 2008-12a disk, we assume $M_{\mathrm{WD}}=$ $1.37 M_{\odot}$ and $R_{\mathrm{WD}}=2000 \mathrm{~km}$, yielding an Eddington limit $\dot{M}_{\text {Edd }}=4 \times 10^{-6} M_{\odot} \mathrm{yr}^{-1}$. We generate a grid of disk models for inclinations $i=10^{\circ}, 20^{\circ}$, and $30^{\circ}$. These models are computed for fixed values of $\dot{M}$ in logarithmic intervals of 0.5 . The true value of $\dot{M}$ is computed by fitting the observed data by interpolating between the computed values of $\dot{M}$. For $R_{0}$, we choose: $=$

$$
R_{0}= \begin{cases}1.0 R_{\mathrm{WD}}, & \dot{M} \leqslant 10^{-7} M_{\odot} \mathrm{yr}^{-1} \\ 1.1 R_{\mathrm{WD}}, & \dot{M}=10^{-6.5} \text { and } 10^{-6} M_{\odot} \mathrm{yr}^{-1} \\ 1.5 R_{\mathrm{WD}}, & \dot{M}=10^{-5.5} M_{\odot} \mathrm{yr}^{-1} \\ 2.0 R_{\mathrm{WD}}, & \dot{M}=10^{-5} M_{\odot} \mathrm{yr}^{-1} .\end{cases}
$$

For M31N 2008-12a, we use Kurucz stellar spectra of appropriate temperature and surface gravity to extend the outer disk to a radius where $3500 \lesssim T<10,000 \mathrm{~K}$. We also consider disks that are truncated in the outer region as discussed in the results section.

Synspec uses the tlusty results for each disk annulus to generate synthetic spectra. These are integrated into a disk spectrum using disksyn, which includes the effects of Keplerian broadening, inclination, and limb darkening (Wade \& Hubeny 1998)

\subsection{Results}

Here, we adopt an inclination of $20^{\circ}$, distance of $770 \mathrm{kpc}$, and reddening $E_{B-V}=0.1$ (DHG17). In Section 4.4, we take the effects of a different inclination $\left(10^{\circ}\right.$ or $\left.30^{\circ}\right)$, an error of

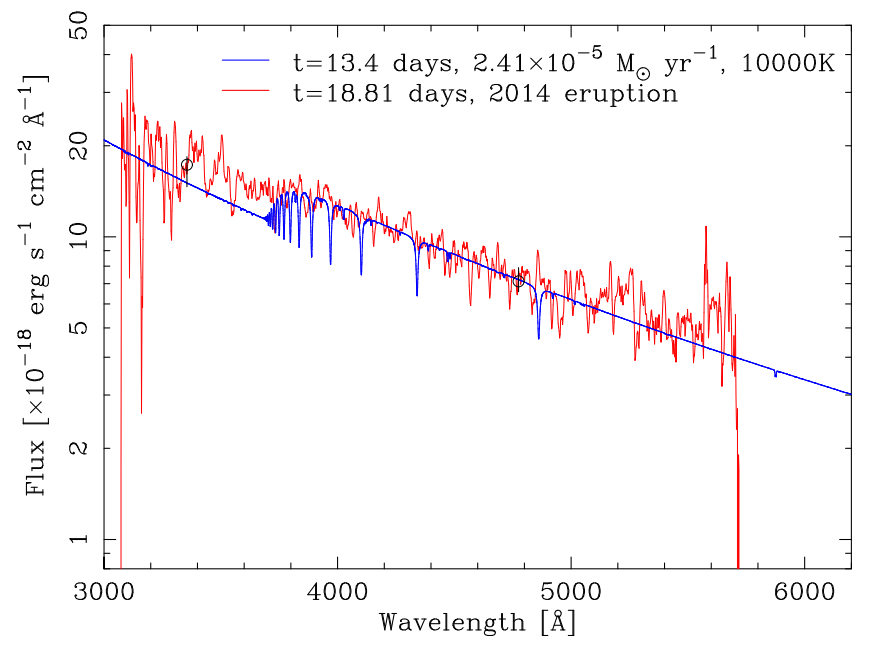

Figure 3. Direct comparison between the $t=13.4$ day accretion disk model of the 2015 eruption and the dereddened $t=18.81$ day Keck spectrum of the 2014 eruption. The flux of the Keck spectrum has been increased by $8.85 \times 10^{-18} \mathrm{erg} \mathrm{s}^{-1} \mathrm{~cm}^{-2} \AA^{-1}$. The spectrum and the model are in good agreement above $4000 \AA$; however, typical accretion disk absorption lines and the Balmer discontinuity are not present in the spectrum, which contains $\mathrm{H} \beta$ in emission.

$\sim 20 \mathrm{kpc}$ on the distance, and a reddening error of 0.03 (DHG17) into consideration and assess how these affect the final results. Since the error bars on the data points are themselves rather small (at most 5\%), they are also considered at the end of this section. A low-inclination system is assumed due to the large UV flux at quiescence and the large observed ejecta velocities (DHB16, DHG17).

The flux data points from the different epochs were obtained through filters covering the given wavelength bands, and as such they represent an average continuum flux level in these regions of the spectrum, possibly also including some prominent lines. One data point (F475W; 4773.7 $\AA$ ) includes $\mathrm{H} \beta$ (which would be in absorption unless there is a disk wind, which we do not model here). We therefore do not expect the data points, at any epoch, to line up nicely with the continuum of the optically thick standard disk model, but rather we use our modeling simply to assess the order of magnitude of the mass accretion rate.

\subsubsection{Quiescence}

We start by modeling the inter-eruption data at $t \simeq 75$ days, as here the flux is at a minimum and we expect the disk to dominate the optical-NUV emission, with negligible contribution from the waning eruption. The modeling at this epoch is then applied, and adjusted as necessary, to the other five epochs.

For the disk models to simply provide sufficient flux to match the observations at the distance of M31, the disk mass accretion rate, ${ }^{22} \dot{M}$, is required to be large, $\gtrsim 10^{-6} M_{\odot} \mathrm{yr}^{-1}$, and therefore not far away from $\dot{M}_{\text {Edd }}$. Such models generate a prominent Balmer discrepancy at $\sim 4000 \AA$, which is not apparent in the quiescent SEDs (also see the late-time spectrum in Figure 3). However, many CVs accreting at a high rate do not exhibit strong Balmer discontinuities (Matthews et al. 2015). We began by fitting the synthetic spectra longward

\footnotetext{
${ }^{22}$ We again note that this may be formally different from the WD mass accretion rate, $\dot{M}_{\text {acc }} \leqslant \dot{M}$.
} 

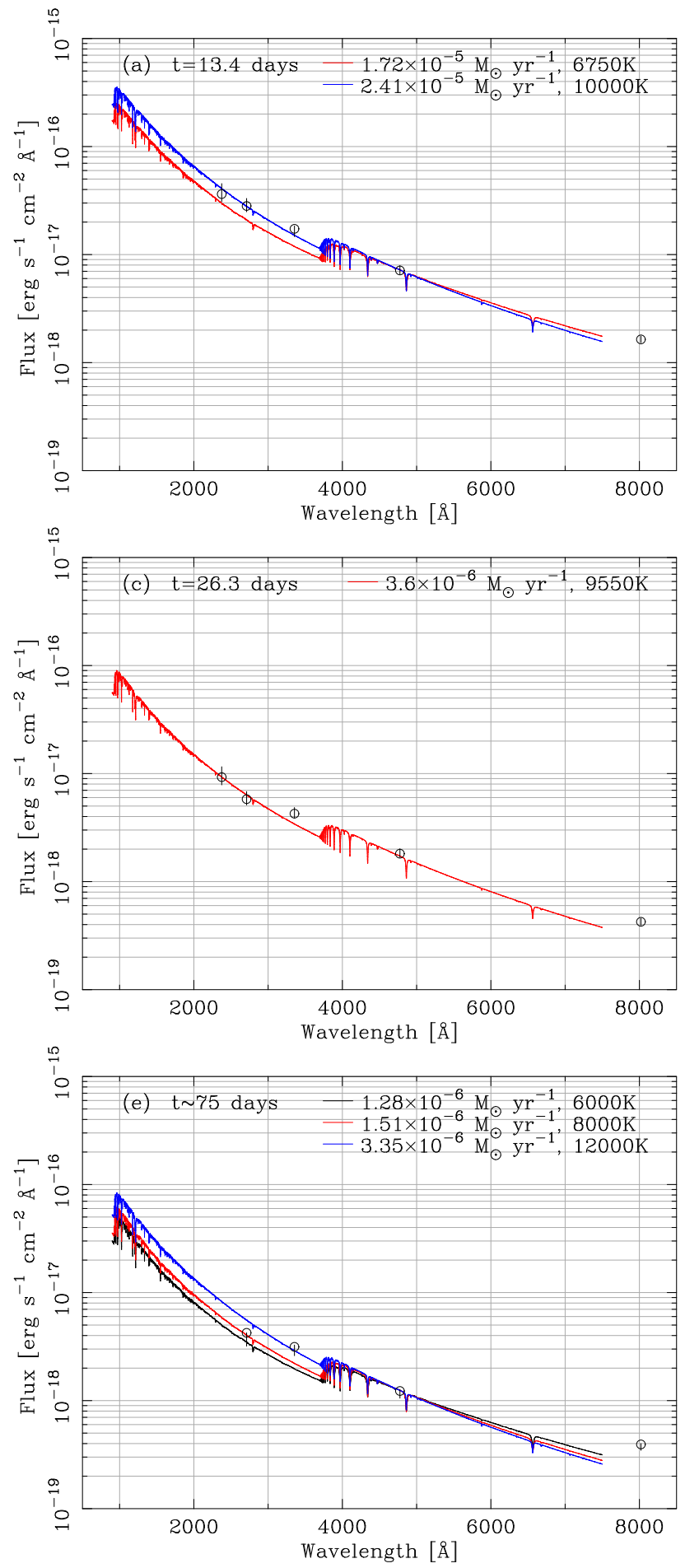
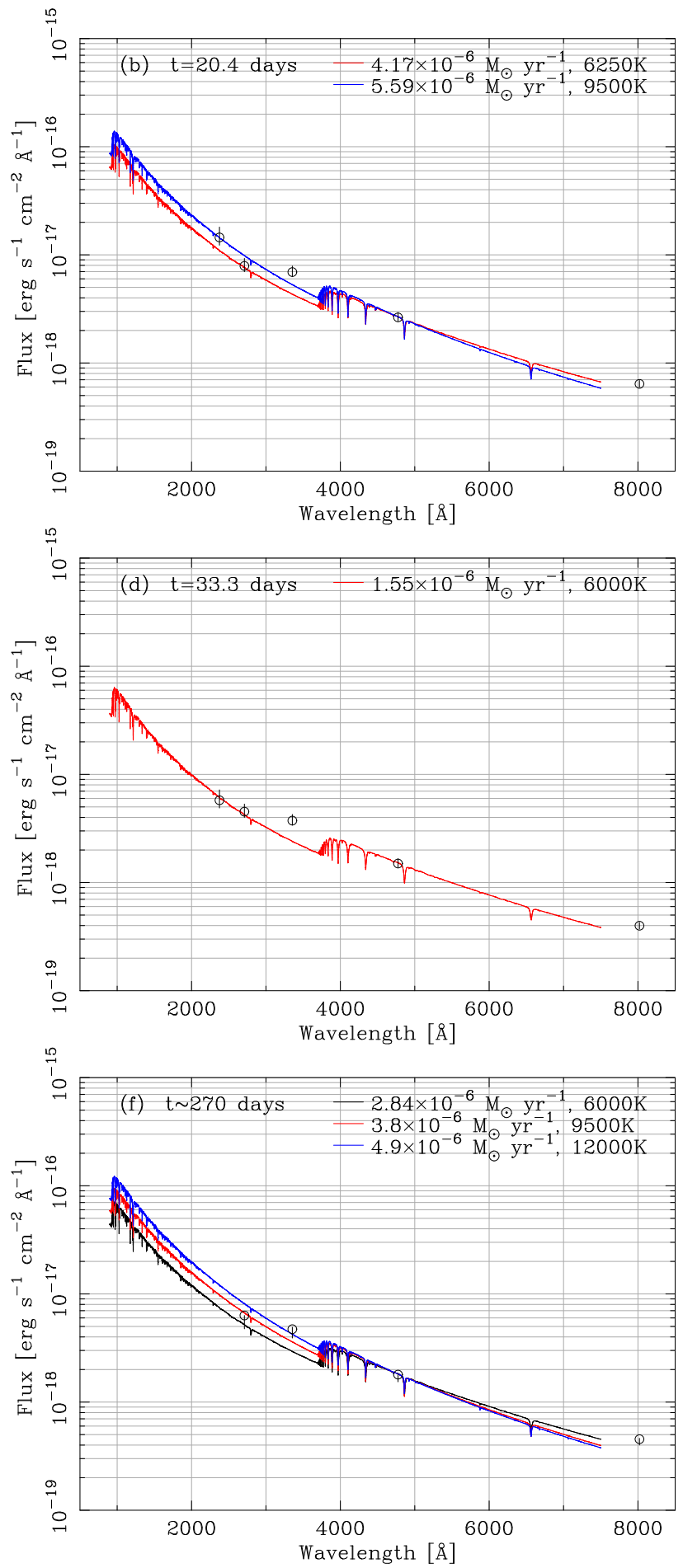

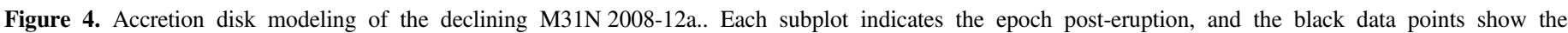

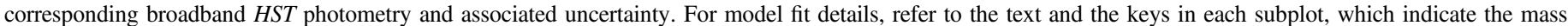

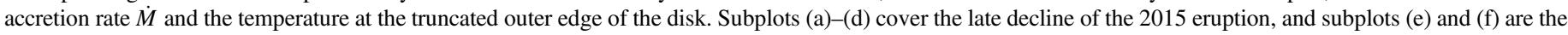
two reconstructed inter-eruption, or quiescent, epochs.

of the Balmer discontinuity to just the $\mathrm{F} 475 \mathrm{~W}$ photometry $(4773.7 \AA)$, which requires $\dot{M}=1.28 \times 10^{-6} M_{\odot} \mathrm{yr}^{-1}$; see Figure 4(e) (solid black line). This model has an outer disk radius extending to $1320 R_{\mathrm{WD}} \simeq 3.8 R_{\odot}$, where the temperature falls to $6000 \mathrm{~K}$, but the model is clearly deficient in flux at wavelengths shorter than the Balmer discontinuity.

Matthews et al. (2015) proposed that the absence or reduction of the Balmer discontinuity observed in some $\mathrm{CVs}$ is due to continuum emission from a disk wind. We note that disk models whose outer radii are truncated also produce spectra with decreased Balmer discontinuities. In Section 5.3, we briefly discuss possible physical explanations for disk truncation. Truncated disk models provide a slightly lower continuum flux level for the same $\dot{M}$.

If the disk is truncated at $R=880 R_{\mathrm{wd}}\left(\approx 2.53 R_{\odot}\right)$, the model fits the first data point (shortest wavelength). This 
Table 5

Computed Accretion Rates for M31N 2008-12a during the Final Decline of the 2015 Eruption and at Quiescence

\begin{tabular}{lccc}
\hline \hline $\begin{array}{l}\text { Epoch } \\
\text { (days) }\end{array}$ & $\begin{array}{c}\dot{M} \\
\left(\times 10^{-6} M_{\odot} \mathrm{yr}^{-1}\right)\end{array}$ & $\begin{array}{c}\dot{M}_{\text {wind }} \simeq \dot{M}_{\text {acc }} \\
\left(\times 10^{-6} M_{\odot} \mathrm{yr}^{-1}\right)\end{array}$ & $\begin{array}{c}\dot{M}_{\text {truncated }} \\
\left(\times 10^{-6} M_{\odot} \mathrm{yr}^{-1}\right)\end{array}$ \\
\hline 13.4 & 17.2 & 8.60 & 24.1 \\
20.4 & 4.17 & 2.86 & 5.59 \\
26.3 & 3.60 & 1.80 & 4.30 \\
33.3 & 1.55 & 0.77 & 1.86 \\
$\sim 75$ & 1.28 & 0.64 & 3.35 \\
$\sim 270$ & 2.84 & 1.42 & 4.90 \\
\hline
\end{tabular}

Note. $\dot{M}$ refers to the disk mass accretion rates as directly calculated by the accretion disk models, $\dot{M}_{\text {truncated }}$ refers to the $\dot{M}$ upper limits imposed by a heavily truncated disk, and $\dot{M}_{\text {wind }}$ is the expected maximum mass loss from the disk via a disk wind, with the remaining amount $\dot{M}_{\text {acc }}$ being accreted onto the WD (i.e., $\dot{M}_{\text {acc }}=\dot{M}-\dot{M}_{\text {wind }}$, or $\dot{M}_{\text {acc, } \min } \simeq \dot{M} / 2$; also see the later discussion about possible outflows).

effectively removes regions of the disk cooler than $8000 \mathrm{~K}$. Outer disk truncation results in a reduced flux (for a fixed $\dot{M}$ ); therefore, the mass accretion rate of this model must rise to $1.51 \times 10^{-6} M_{\odot} \mathrm{yr}^{-1}$ (see Figure 4(e), red line). To fit the second data point, which is nearest to the Balmer edge, we further truncate the disk to $750 R_{\mathrm{WD}}\left(\approx 2.08 R_{\odot}\right)$. Such a disk has $\dot{M}=3.35 \times 10^{-6} M_{\odot} \mathrm{yr}^{-1}$ and the temperature in the outer disk reaches $12,000 \mathrm{~K}$. This model, however, overshoots the first data point. The model is shown in Figure 4(e) (blue line), and the derived accretion rates are tabulated in Table 5. We note that all three models underestimate the F814W flux. The excess flux here may be contributed by the donor (see Sections 4.5 and 5.2). We also note that the differing photometric points at both quiescent epochs were taken at different times; therefore, any fundamental variability at quiescence could be imprinted on these data.

Next, we turn to the second quiescence epoch, $\sim 270$ days post-eruption, and (assuming an annual cycle) $\sim 70$ days preeruption. These data are similar to those at $t \simeq 75$ days (see Figures 4(e) and (f)), but the flux is higher. Consequently, we follow the same modeling procedure. In Figure 4(f), we present three models with the outer disk truncated and $(2.84 \leqslant$ $\dot{M} \leqslant 4.9) \times 10^{-6} M_{\odot} \mathrm{yr}^{-1}$. Again, truncating the cooler outer disk reduces the "jump" of the Balmer edge. From $t \simeq 75$ days to $t \simeq 270$ days, $\dot{M}$ increased by a factor of $\sim 1.5-2.2$.

In all of the models presented here, we found that the inclusion of a hot WD did not contribute any significant flux due to the small surface area of the massive WD and to the very large area of the very hot disk. It is also probable that at high $\dot{M}$, the inner disk is swollen and masks the WD.

\subsubsection{The Decline}

We next consider the evolution during the late decline of the 2015 eruption. We model these in reverse, as the complexity of the emission is expected to increase closer to the eruption itself.

At $t=33.3$ days, the flux from M31N 2008-12a lies approximately midway between that at quiescence $(t \simeq 75$ and $\simeq 270$ days; see the left panel of Figure 2 ), and we find that a standard (non-truncated) disk model with $\dot{M}=$ $1.55 \times 10^{-6} M_{\odot} \mathrm{yr}^{-1}$ provides a reasonable fit to the data. This model has an outer region extending to where the temperature reaches $6000 \mathrm{~K}$; extending the outer region to $3500 \mathrm{~K}$ does not improve the fit to the data points. The fit is presented in
Figure 4(d). There is a slight flux excess at $\sim 3350 \AA$, but as shorter wavelengths are consistent with the model, a truncated disk model does not provide a better fit to the data. Again, there is a flux excess at $\sim 8000 \AA$.

Turning to $t=26.3$ days, the data points are in better agreement with the presence of a weak Balmer edge (see Figure 4(c)). We fit a disk model while varying the outer truncation radius, and find that the best fit is obtained for $\dot{M} \sim 3.6 \times 10^{-6} M_{\odot} \mathrm{yr}^{-1}$ with the outer disk truncated at $9500 \mathrm{~K}\left(R_{\mathrm{disk}}=1050 R_{\mathrm{WD}}, \sim 3 R_{\odot}\right)$.

A week earlier, $t=20.4$ days, we find $\dot{M}=4.17 \times$ $10^{-6} M_{\odot} \mathrm{yr}^{-1}$ and $5.59 \times 10^{-6} M_{\odot} \mathrm{yr}^{-1}$ for disks truncated at $6250 \mathrm{~K}$ and $9500 \mathrm{~K}$, respectively. Neither model reproduces the NUV flux well, possibly an indication of a contribution from an additional source or lines. These two disk models are presented in Figure 4(b).

\subsubsection{The Super-soft X-Ray Phase}

Finally, we turn to the observations at $t=13.4$ days, during the SSS phase of the 2015 eruption. As is evident from Figure 4(a), not only is this the epoch with the highest flux, but the data exhibit a rather smooth "continuum"-almost a straight line on this logarithmic scale. This is further illustrated by the Keck spectrum taken 18.81 days after the 2014 eruption, which is directly compared to the $2015 t=13.4$ day data in Figure 3.

There is no indication of the presence of the Balmer edge from the 2015 data, which is confirmed by the Keck 2014 spectrum. A disk model truncated at $20,000 \mathrm{~K}$ produces a smooth continuum without a Balmer edge, but the continuum slope is much steeper than inferred from the data. Therefore, we fit the data with disk models that have various degrees of truncation. We find $\dot{M}=1.72 \times 10^{-5} M_{\odot} \mathrm{yr}^{-1}$ for a disk truncated at $6750 \mathrm{~K}$ and $2.41 \times 10^{-5} M_{\odot} \mathrm{yr}^{-1}$ for a disk truncated at $10,000 \mathrm{~K}$; see Figure 4(a). As with the other epochs, these models cannot fit all of the data points simultaneously. We note that such an $\dot{M}$ is above $\dot{M}_{\text {Edd }}$.

\subsection{Disk Winds}

As mentioned earlier, Matthews et al. (2015) proposed that the absence or reduction of the Balmer edge in the optical spectra of some CVs is due to the existence of powerful accretion disk winds. Indeed, Matthews et al. (2015) show that a standard disk wind model is successful in reproducing the weak Balmer absorption edge at all inclinations, but particularly for CV systems viewed at high inclination. They further suggest that winds can dominate the continuum emission from CVs. Their modeling shows that the inclusion of the disk wind produces a much weaker Balmer edge and a shallower continuum slope, and the flux level increases due to the contribution of the wind to the disk continuum.

Consequently, compared to the model fit in Matthews et al. (2015), our optically thick non-truncated standard disk models provide an upper limit to the mass accretion rate onto the WD $\left(\dot{M}_{\text {acc }}\right)$, as they produce less flux at the same accretion rate. The discrepancy between the wind disk model and the standard disk model is minimal near the upper edge of the Balmer jump ( 4000-5000 $\AA$ ) and appears to reach a maximum of about a factor of two in $\dot{M}$. Therefore, if we assume that disk wind emission has to be taken into account, we have to reduce the 


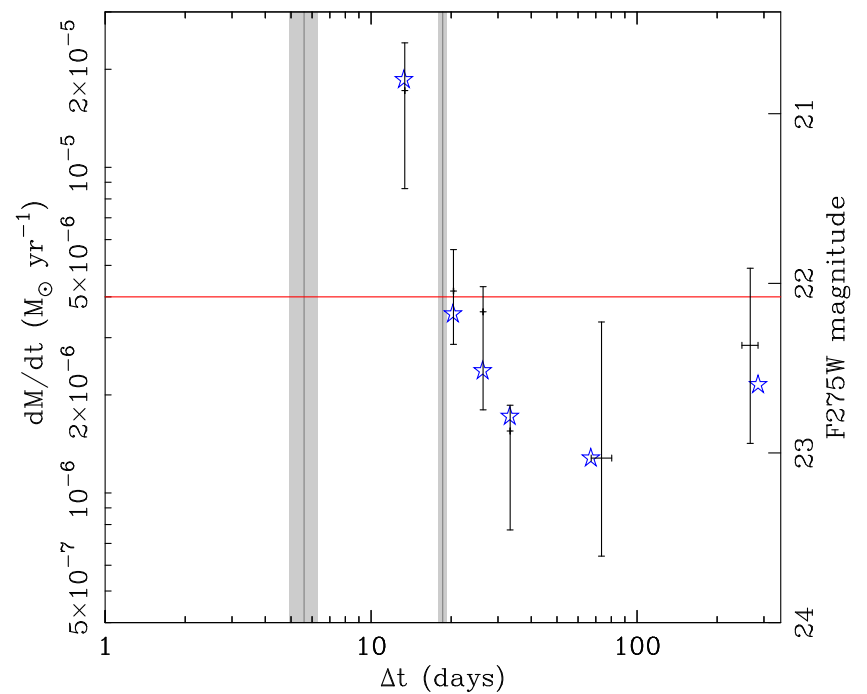

Figure 5. Evolution of the disk mass accretion rate $(\dot{M})$ of the M31N 2008-12a accretion disk with time within an eruption cycle (black points). The upper limits refer to $\dot{M}_{\text {truncated }}$ and the lower limits to $\dot{M}_{\text {wind }}\left(\simeq \dot{M}_{\text {acc }}\right)$; see Table 5. The HST F275W photometry is also plotted for comparison (blue data points) and to indicate that it traces the accretion rate well. The Eddington accretion limit of a $1.37 M_{\odot}$ WD is indicated by the red horizontal line. The vertical gray lines indicate the turn on and turn off times of the SSS from the 2015 eruption (the shaded areas show their associated uncertainties).

mass accretion rates obtained from our non-truncated disk model fits by a maximum of $50 \%$ (i.e., $\dot{M}_{\text {acc }} \simeq \dot{M}_{\text {wind }} \simeq 0.5 \dot{M}$ ). Subsequently, the implied mass accretion rates in the presence of a disk wind are shown in Table 5.

\subsection{Model Uncertainties}

Finally, we compute the relative uncertainties introduced by the errors on the reddening, inclination, distance, and fluxes. For this purpose, we consider the data for $t=33.3$ days, with $\dot{M}=1.55 \times 10^{-6} M_{\odot} \mathrm{yr}^{-1}$.

DHG17 computed that the reddening toward M31N 2008$12 \mathrm{a}$ is $E_{B-V}=0.10 \pm 0.03$. De-reddening the $t=33.3$ day data using $E_{B-V}=0.07$ and $E_{B-V}=0.13$ gives $\dot{M}=1.33 \times$ $10^{-6} M_{\odot} \mathrm{yr}^{-1}$ and $\dot{M}=1.90 \times 10^{-6} M_{\odot} \mathrm{yr}^{-1}$, respectively. That is, the disk mass accretion rate becomes $\dot{M}=$ $1.55_{-0.22}^{+0.35} \times 10^{-6} M_{\odot} \mathrm{yr}^{-1}$.

Similarly, we compute the errors for an inclination of $i=20^{\circ} \pm 10^{\circ}$, distance of $770 \pm 20 \mathrm{kpc}$, and a maximum error of $5 \%$ in the fluxes (see Table 4). Assuming $\dot{M}$ varies linearly with small changes in $E_{B-V}, i, d$ and in the fluxes, by quadrature we obtain $\dot{M}=1.55_{-0.25}^{+0.39} \times 10^{-6} M_{\odot} \mathrm{yr}^{-1}$, giving errors of $+25 \%$ and $-16 \%$. These errors are much smaller than the systematics introduced from the use of a truncated disk model (a factor of $\sim 2$ in $\dot{M}$ ) or when comparing our standard disk models to the Matthews et al. (2015) disk wind models (a factor of $\sim 0.5$ ). Namely, in fitting the data from $t=13.4$ days, we obtained $\dot{M}=1.28 \times 10^{-6} M_{\odot} \mathrm{yr}^{-1}$, but $\dot{M}$ could be about twice this value if we consider the truncated disk models, or it could be about half this value if we consider the possibility of a disk wind continuum.

Taking this into account, we reproduce the computed accretion rates in Table 5 and plot them as a function of time in Figure 5.

\subsection{Donor Flux Excess}

As can be seen in Figure 4, there is a flux excess, above the disk models, in the F814W band at all epochs. As the only expected "red" component in the system, this excess flux is probably from the donor. To examine this effect, we extended the $t \simeq 75$ day and $t \simeq 270$ day accretion disk models to longer wavelengths by fitting a power law to the model spectra, redward of the Balmer limit. This produced a good fit to the model spectra and enabled us to determine an F814W flux excess at quiescence of $(1.67 \pm 0.18) \times 10^{-19} \mathrm{erg} \mathrm{s}^{-1} \mathrm{~cm}^{-2} \AA^{-1}$, which corresponds to an apparent magnitude of $m_{\mathrm{F} 814 \mathrm{~W}}=$ $24.8_{-0.1}^{+0.2}$. Further extrapolation of the accretion disk model confirms that any disk contribution in the NIR F110W and F160W filters is negligible.

\section{Discussion}

\subsection{The Accretion Disk}

In Section 4, we described the comparison between the HST photometry of the final decline of the 2015 eruption and quiescent observations of M31N 2008-12a to models of accretion disks around $1.37 M_{\odot}$ WDs. We again state that the ideal data sets for such work would be spectroscopy extending into (and even beyond) the FUV. However, for CVs at the distance of M31, such observations are not yet feasible. Therefore, the $H S T$ visible and NUV photometry described in this paper currently provide the best, and only, data with which to explore the accretion disk in M31N 2008-12a.

The one thing that is immediately clear is the very large luminosity of the M31N 2008-12a accretion disk at quiescence. By necessity, modeling of such a high-luminosity disk requires a large disk mass accretion rate $(\dot{M})$. The results of the modeling show that the broadband photometric SED of M31N 2008-12a from the epoch of the first post-eruption HST imaging, and during quiescence, is consistent with the expected form of an accretion disk. As the first HST epoch occurs only 13 days after the 2015 eruption, indeed before the SSS is extinguished, this is evidence that the accretion disk may survive eruptions of M31N 2008-12a.

The basic form of the SED, from the optical to NUV, remains consistent from $t=13$ days to quiescence, adding further weight to the survival of the disk. Observationally, we first see this disk beginning to dominate the optical/NUV emission about two weeks post-eruption, and possibly as early as $t=4$ days. $^{23}$ The disk luminosity decreases to a minimum just $\sim 75$ days post-eruption, before building again toward the next eruption-presumably as the accretion disk increases in mass. Our working model is that the disk, once struck by the nova ejecta, is initially shocked and heated, but survives largely intact. Further, irradiation from the SSS may begin to affect the disk from as early as $t=4$ days. These effects cause the disk to begin losing mass at a high rate through a disk wind (with the disk accretion rate at $\dot{M} \sim 2 \times 10^{-5} M_{\odot} \mathrm{yr}^{-1}$ ); as is discussed below, some of this mass may be accreted directly onto the WD. As the surviving disk then cools and relaxes, its luminosity decreases until it reaches a minimum after $\sim 75$ days $\left(\dot{M} \sim 10^{-6} M_{\odot} \mathrm{yr}^{-1}\right)$. During the next $\sim 200$ days of quiescence, mass loss from the donor allows the disk to rebuild any

\footnotetext{
23 Could the optical/NUV light curve plateau presented in DHB16 be caused by the surviving disk being unveiled by the receding photosphere? A similar prediction was made for a number of Galactic RNe by Hachisu et al. (2008).
} 
matter lost (through the eruption and disk wind) in the run up to the next eruption.

But there is a potential problem, not necessarily with the picture outlined above, but with the mass accretion rates derived from the models, which do not include disk winds. Namely, we computed values of $\dot{M}$ representing the disk mass accretion rate, not the accretion rate onto the WD $\left(\dot{M}_{\text {acc }}\right)$. Up to half of $\dot{M}$ might be lost through a disk wind (Matthews et al. 2015), reducing the effective accretion onto the WD by up to $50 \%$. Our disk models imply that $\dot{M}$ is close to, or even exceeds, $\dot{M}_{\text {Edd }}$ throughout the entire eruption cycle, a state where a significant radiation-pressure-driven disk wind may be expected to be present.

A number of authors have investigated the WD mass-WD accretion rate $\left(\dot{M}_{\text {acc }}\right)$ phase space, and they arrive at two broad but differing conclusions. The first is that, other than $\dot{M}_{\text {Edd }}$ itself, there is (for a given WD mass) no upper limit on the mass accretion rate, and that nova eruptions will occur at any $\dot{M}_{\text {acc }}$ (see, e.g., Starrfield 2016). Or alternatively, that there is a clear upper limit to $\dot{M}_{\text {acc }}$ (see, e.g., Fujimoto 1982; Nomoto 1982), beyond which nova eruptions cease, with the WD entering a phase of steady-state nuclear burning (the persistent SSS). At even higher accretion rates, these models predict that optically thick winds (from the WD) are generated. In recent years, it has been proposed that one important difference between these two scenarios is how mass accretion is treated during a nova eruption (Hachisu et al. 2016), with the former assuming it ceases, and the latter assuming it continues. With M31N 2008-12a showing both signs of a surviving disk, therefore continuing accretion, and an elevated $\dot{M}$, it may be an important system in addressing this long-standing issue.

Discussion of the merits of these two differing pictures is clearly beyond the scope of this paper. But we note, of course, that the former (with no upper limit) poses no clear obstacle to our derived accretion rates. Turning to the latter, we note that the work of Kato et al. (2014, 2015, 2016, 2017a, 2017b), employing such a formulation, has already successfully modeled many observational aspects of the M31N 2008-12a eruptions, while assuming a constant $\dot{M}_{\text {acc }}=1.6 \times 10^{-7} M_{\odot} \mathrm{yr}^{-1}$ - a factor of four lower than the $\dot{M}_{\text {acc }}$ lower limit derived in this work (under the assumption of $\dot{M}_{\text {acc }} \simeq \dot{M}_{\text {wind }}$ ). As is shown graphically in Figure 6, accretion disks with $\dot{M}=1.6 \times$ $10^{-7} M_{\odot} \mathrm{yr}^{-1}$ significantly underpredict the NUV flux of M31N 2008-12a at quiescence. The discrepancy is a factor of $\sim 10$, even at the quiescence minimum of $\sim 75$ days posteruption.

In Figure 7, we have recreated Figure 6 of Kato et al. (2014; M. Kato 2017, private communication), which shows the loci of equi-recurrence periods of novae in the WD mass- $\dot{M}_{\text {acc }}$ plane. In this plot, as discussed above, the regions of proposed steady burning and optically thick winds are shown. The position of M31N 2008-12a as computed by Kato et al. (2014) is indicated by the red star, and this lies clearly at the extremes of the phase space permitted by these models. The disk mass accretion rates computed in this work are clearly at odds with the Kato et al. (2014) formulation, unless only a small proportion of the matter from the disk is accumulated on the WD surface. Given our computed mass-loss rates, we would require at least $80 \%$ of $\dot{M}$ to constitute a disk wind $\left(\dot{M}_{\text {wind }}\right)$ to stop the system from undergoing the proposed steady-state nuclear burning. However, such an elevated $\dot{M}_{\text {wind }}$ seems

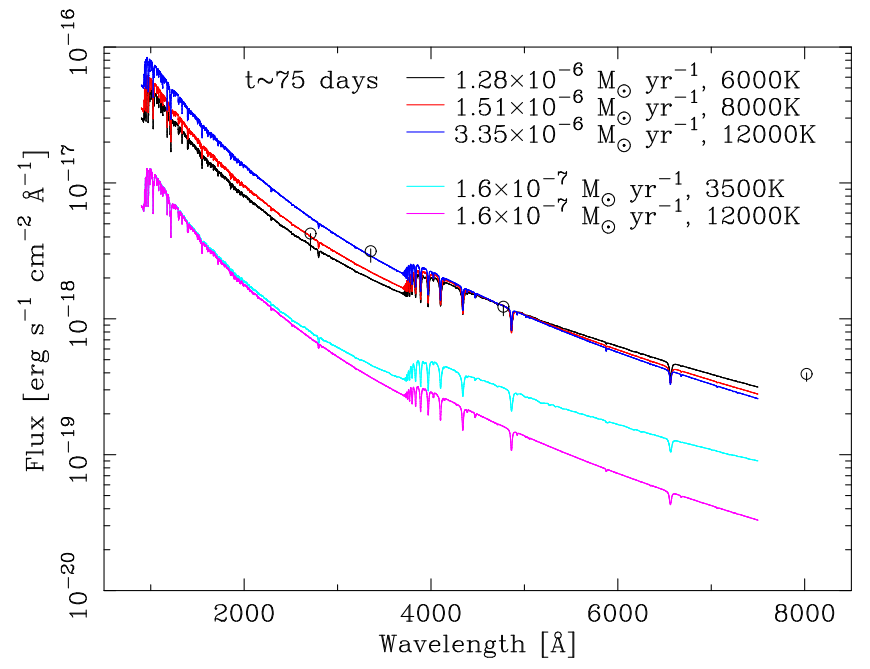

Figure 6. As in Figure 4, comparing the best-fit accretion disk models to the HST photometry $\sim 75$ days post-eruption-the minimum state. Also shown, in magenta and cyan, are our predicted accretion disk spectra based on an accretion rate of $\dot{M}=1.6 \times 10^{-7} M_{\odot} \mathrm{yr}^{-1}$, as required by Kato et al. (2014, 2015, 2016, 2017b). Disks with the Kato et al. accretion rate underpredict the quiescent flux of M31N 2008-12a by a factor of $\sim 10$.

unlikely for a subcritical accretion disk (see, e.g., Poutanen et al. 2007).

Since our disk model indicates such a large $\dot{M}$, we must explore the validity of the standard $\alpha /$ Shakura \& Sunyaev (1973) disk model as $\dot{M}$ approaches $\dot{M}_{\text {Edd }}$, since the basic "geometrically thin" assumption breaks down, i.e., height/ radius $\approx 1$. In this regime of $\dot{M} \approx \dot{M}_{\text {Edd }}$, the disk can be represented using the slim-disk equations (Abramowicz et al. 1988), where radial advection and radiation of energy are taken into account, and the flow can be partially supported by gas and radiation pressure. The departure from the standard disk model, however, is noticeable at mass accretion rates reaching $\dot{M} \sim 20 \dot{M}_{\text {Edd }}$ (Abramowicz et al. 1988) as the heat trapped within the matter becomes important, and the luminosity increases more slowly than the accretion rate as the matter with its energy content is advected and radiated inward to the inner disk and onto the WD surface. Since the maximum mass accretion we compute in this work is $\dot{M} \approx$ $4 \dot{M}_{\text {Edd }}<20 \dot{M}_{\text {Edd }}$ and the minimum is as low as $0.2 \dot{M}_{\text {Edd }}$ during quiescence, our disk models are probably not strongly affected by neglecting the advection of energy.

Advection of energy is, however, more pronounced in the inner disk and can be expected to peak in the boundary layer between the WD and disk, since an additional $L_{\mathrm{bl}} \approx L_{\mathrm{acc}} / 2$ is released in that region. This does not affect our disk models either, as the inner annuli in our models do not contribute significant flux at wavelengths $>2000 \AA$ because of their small surface area $\left(r<6.5 R_{\mathrm{WD}}\right)$ and elevated temperature $\left(>3 \times 10^{5}\right.$ $\mathrm{K})$ peaking in the EUV/soft X-ray regime $\left(\sim 10^{5} \mathrm{~K}\right)$.

Having established that our disk models are valid, we furthermore consider the fate of the advected energy in the inner disk/boundary layer. It has been shown that even at moderately large accretion rates $\left(\dot{M} \approx \dot{M}_{\text {Edd }}\right)$, the advection of energy becomes important in the boundary layer (Godon 1997; Popham 1997). As in advection-dominated accretion flows (ADAFs; Narayan \& Yi 1994, 1995), the inner disk and boundary layer will radiate significantly less than expected, and the advected energy will heat up the WD and drive a bipolar outflow $\left(\dot{M}_{\mathrm{bl}}\right)$ in addition to the disk wind component. This will 


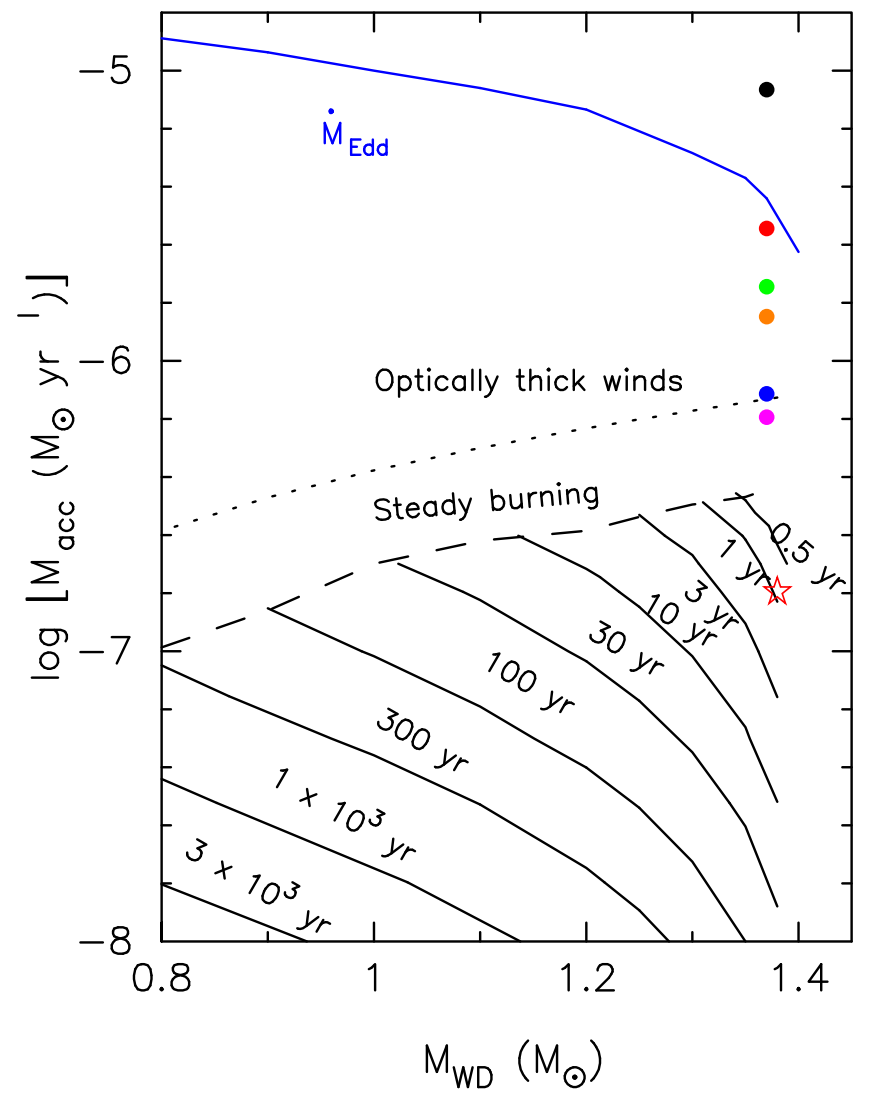

Figure 7. Recurrence period $t_{\mathrm{rec}}$ of novae on the $M_{\mathrm{WD}}-\dot{M}_{\text {acc }}$ plane, based on Figure 6 of Kato et al. (2014). We plot the loci of the equi-recurrence periods of novae (black solid lines). Hydrogen burning is proposed to be stable in the region above the dashed line $\left(\dot{M}_{\text {stable }}\right)$. In the region below $\dot{M}_{\text {stable }}, H$-shell burning is thermally unstable, and the WD experiences shell flashes (i.e., novae). Optically thick winds are accelerated in the region above the dotted line $\left(\dot{M}_{\text {cr }}\right)$. We note that the Starrfield et al. (2016) interpretation of this plot requires neither steady burning nor optically thick wind regions (see the text for more details). The solid blue line indicates the Eddington limit (based on Nomoto 1982). The red star indicates the quiescent position of M31N 2008$12 \mathrm{a}$, based on the modeling in Kato et al. (2015). The colored points, at $M_{\mathrm{WD}}=1.37 M_{\odot}$, indicate the lower limits of the computed values of $\dot{M}$ (i.e., assuming that $\dot{M}_{\text {acc }}=0.5 \dot{M}$ ) from (top-bottom) $t=13.4$ (black), 20.4 (red), 26.3 (green), $\sim 270$ (orange), 33.3 (blue), to $\sim 75$ days (magenta) post-eruption (see the $\dot{M}_{\text {wind }} \simeq \dot{M}_{\text {acc }}$ values in Table 5).

reduce the amount of material actually accreting onto the WD surface and could bring the WD accretion rate back toward the regime favored by Kato et al. (2015) and others. The outflow of matter is possibly low in the outer disk and increases inward, where a strong wind forms a bipolar outflow.

For a number of decades, some CVs have been suspected to have strong outflows, with some systems even exhibiting ejecta, such as the "nova-like" BZCamelopardalis that is surrounded by a bow-shock nebula (Ellis et al. 1984). However, so far, one finds no collimated outflows ("jets") in CVs (Hillwig et al. 2004) despite the fact that all other disk systems (from X-ray binaries to AGNs) exhibit collimated outflows (Livio et al. 1997). Is it possible that M31N 2008-12a is the exception to the rule, not just during eruption (see DHB16 and DHG17) but at quiescence?

If we assume a strong disk wind, then about half of the disk material is accreted onto the WD (at a rate of $\dot{M}_{\text {acc }} \sim$ $6.4 \times 10^{-7} M_{\odot} \mathrm{yr}^{-1}$ at the apparent quiescent minimum) and the other half is deposited into the system (at the same rate; see Table 5 and Section 5.2) — the circumbinary environment. We note that the estimated red giant wind mass (total) in RS Oph at the time of the eruption is $\sim 10^{-6} M_{\odot}$ (Vaytet et al. 2011), broadly consistent with the circumbinary contamination predicted by the M31N 2008-12a disk wind. Therefore, such a disk wind mass-loss rate alone could be sufficient to account for the observed ejecta deceleration (DHB16) without a requirement for a wind from the donor.

The discussion of the accretion disk would not be complete without considering the irradiation of the outer disk by the hot inner disk/boundary layer region. Disk irradiation is known to be important in low-mass X-ray binaries, where accretion occurs onto a neutron star or a black hole, with a much deeper gravitational potential well, while it is usually negligible in CVs (van Paradijs \& McClintock 1994; Shahbaz \& Kuulkers 1998; King 1998). However, the WD in M31N 2008-12a is very compact with a mass of $1.37 M_{\odot}$ and a radius of $R_{\mathrm{WD}}=2000 \mathrm{~km}$, and it is accreting at, or close to, the Eddington limit. We therefore checked the importance of disk irradiation using the approach given by Vrtilek et al. (1990) for different values of $\dot{M}$. At low disk mass accretion rates $\left(\dot{M} \lesssim 10^{-7} M_{\odot} \mathrm{yr}^{-1} ;\right.$ as is typical for all other quiescent novae), irradiation increases the outer disk temperature by up to $\sim 1000 \mathrm{~K}$, which does not produce any significant change in the disk spectrum. At more moderate accretion rates $\left(\dot{M} \sim 10^{-6} M_{\odot} \mathrm{yr}^{-1}\right.$; i.e., M31N 2008-12a at quiescence), irradiation increases the outer disk temperature by up to $\sim 3000 \mathrm{~K}$, thereby slightly affecting the spectrum by effectively decreasing the mass accretion rate, since irradiation increases the disk emission. At mass accretion rates above the Eddington limit $\left(\dot{M} \sim 10^{-5} M_{\odot} \mathrm{yr}^{-1}\right.$; M31N 2008-12a during eruption), we find that irradiation increases the outer disk temperature by as much as $7000 \mathrm{~K}$, and we would expect that this increase could reduce the mass accretion rate by a factor of $\sim 2$. The effect of irradiation within M31N 2008-12a at quiescence is therefore only expected to slightly decrease the discrepancy in the disk mass accretion rate and the WD mass accretion rate.

We return finally to the survival of the accretion disk. The presence of a disk, potentially with a high mass accretion rate, during the SSS phase of a nova eruption opens up an intriguing possibility. Could a surviving accretion disk continue to feed significant fuel to the nuclear burning region on the WD, thereby "artificially" extending the SSS phase, compared to a more typical nova (where the disk is assumed to be obliterated by the eruption)? Such a "refuelling" would, for a short time, be akin to the persistent SSSs. Any mass accreted onto the WD during this period would be burned to He and simply be added to the mass of the WD. Irrespective of the net gain or loss of accumulated mass during the nova eruption, "refuelling" would enable net WD mass growth over the refuelling period. Here we only offer an outline of the concept, as this is explored in more detail, observationally and theoretically, in Henze et al. (2017).

\subsection{The Donor}

There is an expectation that NIR observations of a quiescent nova system will largely isolate the donor star (see Darnley et al. 2012 and Figure 2), particularly for evolved (i.e., luminous) donors. The accretion disk models employed in this work only extend to $7500 \AA$, but a simple extrapolation to longer wavelengths confirms that we can expect little, or no, accretion contribution to the quiescent flux in the NIR regime. Therefore, we conclude that the PHAT NIR quiescent 

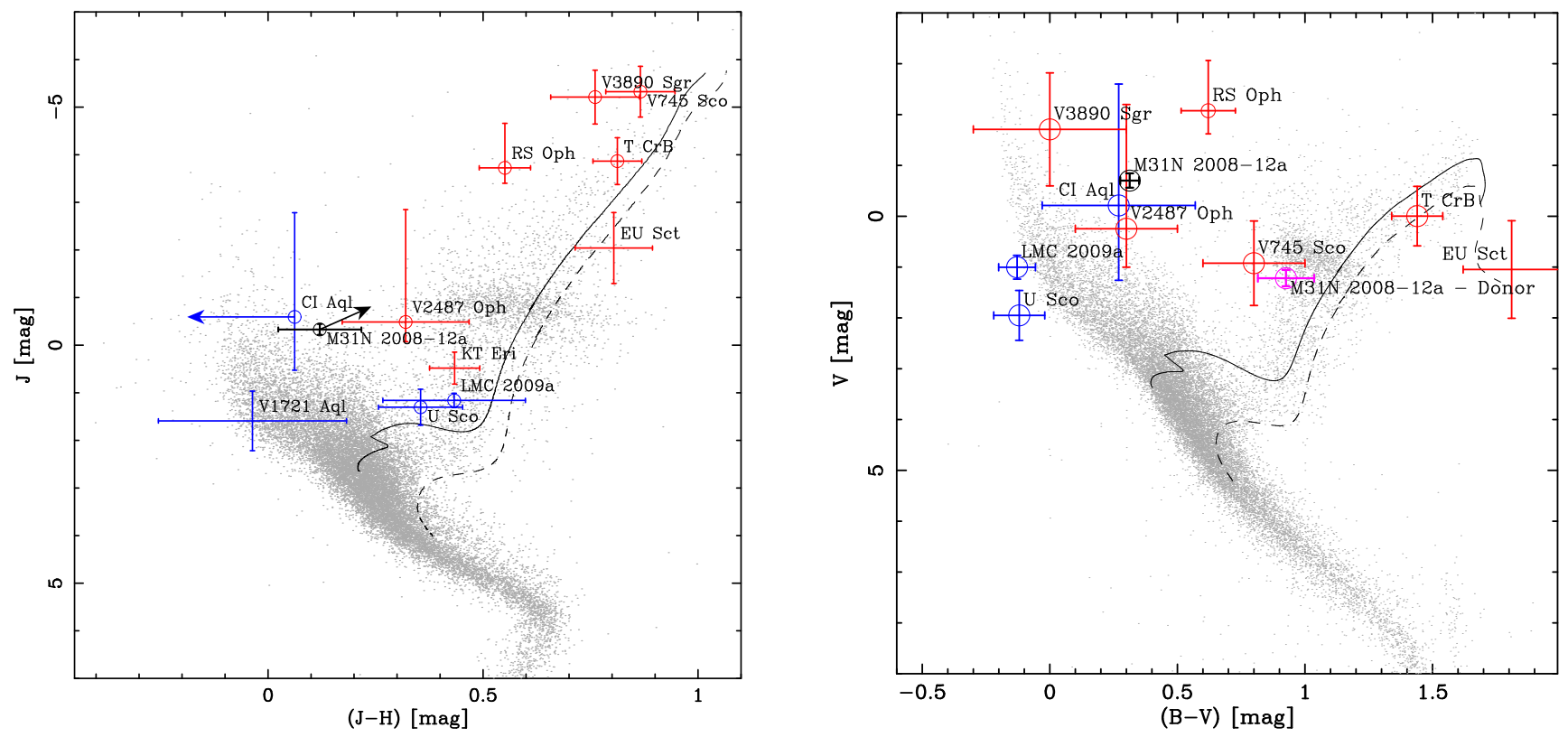

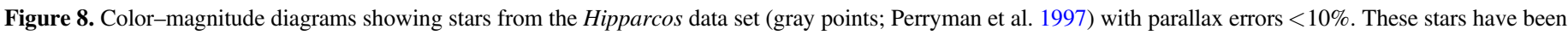

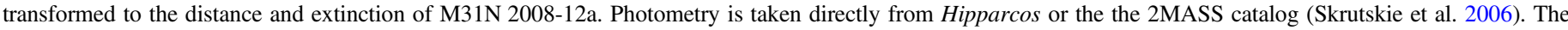

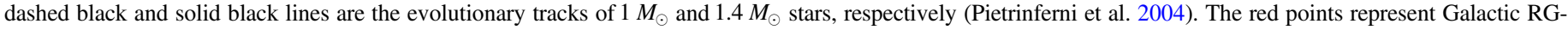

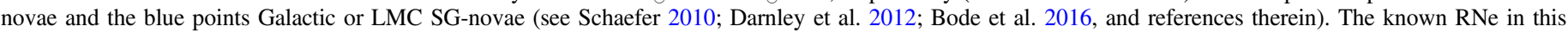

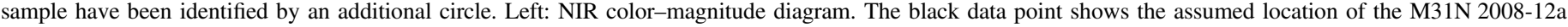

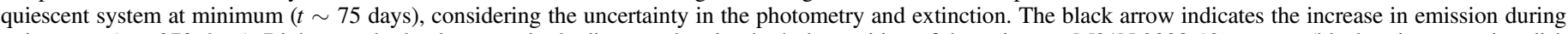

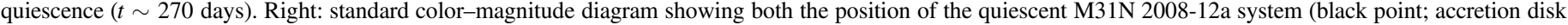
+ donor; $t \sim 75$ days) and the inferred position of the mass donor alone (magenta), which is consistent with that of the M31 red clump (see Section 5.2).

photometry should simply be the photometry of the M31N 2008-12a mass donor. In Section 4.5, we used the accretion disk modeling at quiescence to estimate the $I$-band $(\mathrm{F} 814 \mathrm{~W})$ contribution from the donor.

As can be seen in the left plot of Figure 8, the position of the quiescent M31N 2008-12a on an NIR color-magnitude diagram indicates that the donor is significantly less luminous and bluer than the red giants contained in the Galactic RG-novae (red points). However, the M31N 2008-12a donor may be consistent with the M31 red clump. A simple blackbody fit to the F110W $(\sim J)$ and F160W $(\sim H)$ photometry at quiescence ( $t \sim 75$ days $)$ yields $L_{\text {donor }} \sim 100 L_{\odot}$ and $T_{\text {eff,donor }} \sim 4800 \mathrm{~K}$.

If we include the extrapolated $I$-band luminosity of the donor (see Section 4.5), then the three-point donor SED is very well-represented by the same blackbody fit. Hence, we find that the M31N2008-12a donor may be consistent with a blackbody of $T_{\text {eff,donor }}=4890 \pm 110 \mathrm{~K}, L_{\text {donor }}=103_{-11}^{+12} L_{\odot}$, and $R_{\text {donor }}=14.14_{-0.47}^{+0.46} R_{\odot}$, at the distance of M31. This blackbody fit is illustrated by the dashed black line in the righthand plot of Figure 2. We note that the quoted uncertainties are the formal $1 \sigma$ errors resulting from the fitting process; the effects of possible systematic uncertainties related to the accretion and blackbody disk modeling have not been estimated. Using this blackbody, we compute the extrapolated $B$ and $V$ photometry (again indicating the above caveats) of the donor and plot its position on a standard color-magnitude diagram in the right plot of Figure 8 . Here, it is clear that the donor may indeed be consistent with the M31 red clump.

As is illustrated in the left plot of Figure 8, as the quiescent system evolves from its minimum state ( $t \sim 75$ days) toward the next eruption $(t \sim 270$ days $)$, the donor brightens by $J \sim 1$ mag, but becomes redder-consistent with an increase in the donor radius. Such behavior may be related to irradiation of the donor, causing heating and expansion of the atmosphere. It could also be related to the orbital phase of the system, with a tidally locked donor, for example, being non-spherically symmetric and unevenly heated-but such phase effects would imply a high system inclination. The very high luminosity of the accretion disk almost certainly now rules out high inclinations.

Given the available evidence, we must conclude that the mass donor in the M31N 2008-12a system is either a (low luminosity) red giant or a post-red giant branch star (e.g., horizontal branch), and/or that it is affected by significant irradiation from the primary, the disk, and the eruptions. Of course, we must point out that there is a possibility that the star identified as the donor may simply be another star at a very similar position on the sky within M31. If we were relying on WFC/IR photometry of the donor alone, this probability would be quite large, but given the F814W spatial resolution of $H S T$, the likelihood will be relatively small (around 2\%; see DWB14 and Williams et al. 2014b).

One piece of evidence may be key to constraining the donor, however. DHS15 proposed that the M31N 2008-12a ejecta interact strongly, and immediately, with material in the circumbinary medium with a $1 / r^{2}$ density dependence. This picture was strengthened by the reanalysis presented in DHB16. With such behavior being seen consistently across four consecutive eruptions (2012-2015), the circumbinary material must be continuously replenished. One feasible source of such material seems to be a stellar wind from a red giant donor as observed in RS Oph (see e.g., Bode et al. 2006). As ejecta-circumbinary shocks are not observed in $\mathrm{CNe}$, we must infer that Roche lobe overflow is too efficient a mass transfer process to build up significant material in the circumbinary 
environment. Therefore, the donor would be strongly constrained to any star capable of generating such a wind.

However, in this paper, we explored the possibility that the extremely luminous accretion disk generates a significant disk wind. Therefore, is it possible that such a disk wind is the source of the circumbinary pollution, not the stellar wind of a red giant donor? As such, a giant donor could be transferring matter at a high rate to the disk via Roche lobe overflow. Indeed, could such a scenario be the only feasible manner in which such a high sustained WD mass accretion rate could be achieved?

\subsection{Orbital Period}

To date, the orbital period of the M31N 2008-12a system has eluded observation. However, given we now know the mass of the WD and have constrained the radius of the donor, we can place some restrictions on $P_{\text {orb }}$. We will assume that the donor has evolved at least enough to reside on the red giant branch and that it was originally the lower-mass component of the binary. Therefore, the donor mass must be somewhere in the range $0.8-8 M_{\odot} \cdot{ }^{24}$ If we assume that the donor is Roche lobe filling, then the orbital separation must be in the range $25-44 R_{\odot}$ $(0.12-0.20 \mathrm{au})$, hence $5 \lesssim P_{\text {orb }} \lesssim 23$ days. If the accretion is stellar wind driven, then $P_{\text {orb }} \gg 5$ days. We note that such minimum orbital separations, and hence Roche lobe sizes, are far too large to account for natural accretion disk truncation by the presence of the donor.

Based on a suspected red giant donor, DHB16 suggested that M31N 2008-12a might be the only known nova with $P_{\text {rec }}<P_{\text {orb }}$. Therefore, we again point out that as $\dot{M}$ is a function of the donor-WD separation, that if $P_{\text {rec }}<P_{\text {orb }}$, any orbital eccentricity may affect the accretion rate and inter-eruption timescale on an eruption by eruption basis.

\subsection{The X-Ray Flash Non-detection}

The production of an X-ray flash at the onset of a nova eruption is a long-standing prediction (Starrfield et al. 1990; Krautter 2002). Kato et al. (2016) reported the results of an intensive Swift observing campaign to detect any X-ray flash associated with the 2015 eruption of M31N 2008-12a. This campaign did not detect such a flash, and Kato et al. (2016) presented two explanations for the non-detection. First, the $\mathrm{X}$-ray flash simply occurred before the Swift monitoring began - which requires the X-ray flash to precede the optical/NUV nova by $\gtrsim 8$ days. The second proposed that significant circumbinary material masked the flash signature. At the time, with little firm evidence for the nature of the donor, Kato et al. (2016) preferred the first explanation. However, given the work reported in this paper, we emphasize that the X-ray flash could have been missed due to significant absorption from circumbinary material. This material could consist of some combination of a donor wind and a disk wind. For a low-inclination system with a significant disk wind, the bulk of the circumbinary material could even reside along the line of sight. But the material in a disk wind dominated scenario may be expected to already be highly ionized, and hence unlikely to be able to mask any flash. Therefore, the X-ray flash could have been absorbed if there was significant pollution of the

\footnotetext{
24 The lower limit to allow evolution onto the red giant branch by the present day; the upper limit is the approximate zero-age upper mass limit to form a WD.
}

circumbinary environment by the wind of the donor, but likely only if the donor is not Roche lobe filling.

\subsection{Time to Reach the Chandrasekhar Mass}

TBW14 presented a prediction of the time required for the WD within the M31N 2008-12a system to grow to the Chandrasekhar mass (or at least to $1.37 M_{\odot}$ ). For example, they presented a $1.36 M_{\odot}$ WD, with $\dot{M}_{\text {acc }}=1.7 \times$ $10^{-7} M_{\odot} \mathrm{yr}^{-1}$, and a mass retention rate (the amount of accreted material remaining on the WD surface post-eruption) of $35 \%$. This resulted in a timescale to grow to the Chandrasekhar mass of $\sim 200 \mathrm{kyr}$.

We can update this simple calculation using the results from this paper and from DHS15, but using the same approach as TBW14. We will assume that the WD mass is actually $1.37 M_{\odot}$, as used for the disk modeling, and that a further $0.01 M_{\odot}$ of accretion is required to reach the Chandrasekhar mass (the same required mass growth as in TBW14). HND15 determined that $(2.6 \pm 0.4) \times 10^{-8} M_{\odot}$ of $\mathrm{H}$ is ejected in each eruption - as a conservative estimate, we will therefore assume that the total ejected mass is $6 \times 10^{-8} M_{\odot}{ }^{25}$ (consistent with Kato et al. 2015). ${ }^{26}$ Retaining this conservative stance, we will assume that the average WD mass accretion rate over the entire 1 year cycle is in fact the absolute minimum rate predicted by this paper, $\dot{M}_{\text {acc }}=6.4 \times 10^{-7} M_{\odot} \mathrm{yr}^{-1}$, assuming a similar amount of mass is lost in the form of a disk wind. Even then, at such a lower accretion limit, the retained mass, or accretion efficiency, is a staggeringly high $90 \%$. This is much higher than the $\sim 30 \%$ predicted by TBW14 and the $63 \%$ calculated by Kato et al. (2015). Combining these new data, we arrive at an updated prediction of the time to reach the Chandrasekhar mass of $<20 \mathrm{kyr}$ - and possibly much shorter.

\section{Summary and Conclusions}

In this paper, we presented our analysis of an unrivaled series of HST photometric observations of the final stages of the 2015 eruption of M31N 2008-12a. In this analysis, we also exploited archival HST imaging during quiescence and the Keck spectroscopy of the 2014 eruption of M31N 2008-12a. Our main findings include the following.

1. The HST WFC3/UVIS photometry of the late decline of the 2015 eruption shows a steady decline toward quiescence from the $\sim I$-band to the NUV, broadly consistent with the general trends established from the early-decline ground-based and Swift photometry.

2. When combined with the archival HST photometrywhich is shown to have been taken between eruptionsthe system appears to reach a flux minimum (in all bands) $\sim 75$ days post-eruption, before again increasing in luminosity by $\sim 270$ days post-eruption, $\sim 100$ days before the next eruption.

3. The broadband SEDs of the late decline and during quiescence were explored using accretion disk models. The results indicate that these SEDs, even as early as 13.4 days post-eruption, are consistent with the emission

\footnotetext{
25 Assuming roughly equal masses of $\mathrm{H}$ and $\mathrm{He}$ in the ejecta. Kato et al. (2014) assume $X=0.55, Y=0.43$, and $Z=0.02$ in the ejecta.

${ }^{26}$ We further note that this ejected mass assumes a spherical geometry. With highly asymmetrical ejecta and the proposed low inclination, it is possible that the ejected mass is much greater.
} 
being dominated by an accretion disk-one that has survived the eruption.

4. The inferred accretion rates are initially above the Eddington accretion limit $(t=13.4$ days), indicating a disk that has survived, albeit in a severely shocked and heated state. The disk luminosity and inferred $\dot{M}$ then decline toward minimum ( $t \sim 75$ days) before increasing again ( $t \sim 270$ days), presumably as the disk fully re-establishes.

5. The computed accretion rates, even at quiescence, are large, with the disk luminosities still close to the Eddington limit. We speculate that mass loss from the disk will lead to a disk wind.

6. Could such a disk wind contribute a significant quantity of material to the circumbinary environment, and could it provide the matter source with which the ejecta are observed to interact, and possibly even the ejecta collimation mechanism?

7. Our disk modeling computed a range of $\dot{M}=$ (1.2-2.8) $\times 10^{-6} M_{\odot} \mathrm{yr}^{-1}$ during quiescence. Even when accounting for disk winds, which might account for half of $\dot{M}$, the derived accretion rates onto the WD at quiescence are still in the range $\dot{M}_{\text {acc }}=(0.6-1.4) \times$ $10^{-6} M_{\odot} \mathrm{yr}^{-1}$, significantly larger than any other nova. If confirmed, WD accretion rates this high will cause significant problems for a number of well-established nova eruption models.

8. DHB16 and DHG17 both proposed the presence of highly collimated outflows or even jets during the eruption. Could such a high $\dot{M}$ drive a bipolar outflow from the inner disk and boundary layer, even at quiescence?

9. Archival $H S T$ WFC3/IR photometry on the system isolates the donor. Coupled with a strong $\sim I$-band excess from the accretion disk modeling, this photometry indicates a donor with $T_{\text {eff,donor }}=4890 \pm 110 \mathrm{~K}, L_{\text {donor }}=103_{-11}^{+12} L_{\odot}$, and $R_{\text {donor }}=14.14_{-0.47}^{+0.46} R_{\odot}$ - consistent with the M31 red clump.

10. The NIR colors of the donor are slightly redward of the red clump, and there is significant variation in the donor luminosity at quiescence. These may be signs that the donor is significantly irradiated by the WD, disk, and ejecta, or may also be due to orbital phase effects.

11. Based on the work presented in this paper, the updated timescale for the system to reach the Chandrasekhar mass has fallen to $<20 \mathrm{kyr}$.

These HST observations of the late-decline of the 2015 eruption, combined with serendipitous archival detections during quiescence, have started to shed some light on the inter-eruption behavior of M31N 2008-12a. It is clear that UV observations of this remarkable system are key to fully untangling the extreme physics at play throughout the entire eruption cycle. Vital questions that should be addressed over the coming eruptions include the balance between accreted matter and ejected matter, in light of the apparent large variation in the quiescent $\dot{M}$, to fully assess the ultimate fate of M31N 2008-12a.

We would first like to express our gratitude to the anonymous referee for their detailed comments and suggestions, which helped improve the content and readability of the manuscript.
Based on observations made with the NASA/ESA Hubble Space Telescope, obtained from the Data Archive at the Space Telescope Science Institute, which is operated by the Association of Universities for Research in Astronomy, Inc., under NASA contract NAS 5-26555. These observations are associated with programs \#12056, \#12106, and \#14125.

The W. M. Keck Observatory is operated as a scientific partnership among the California Institute of Technology, the University of California, and the National Aeronautics and Space Administration. The Observatory was made possible by the generous financial support of the W. M. Keck Foundation. The authors wish to recognize the significant cultural role that the summit of Maunakea has always had within the indigenous Hawai'ian community. We are most fortunate to have the opportunity to conduct observations from this mountain.

This research has made use of the Keck Observatory Archive (KOA), which is operated by the W. M. Keck Observatory and the NASA Exoplanet Science Institute (NExScI), under contract with the National Aeronautics and Space Administration. The authors also acknowledge Sumin Tang as the PI of program C204LA. We also acknowledge the Keck observer Adam Miller.

The authors would like to thank the HST staff for their heroic efforts scheduling our early-time, and disruptive, spectroscopic observations. Particular thanks are given to Charles R. Proffitt, the STScI Contact Scientist for programme \#14125, for his support with the STIS observations.

M.J.D. would like to personally thank Mariko Kato for providing Figure 7, and Christian Knigge and James Matthews for discussions on accretion disk winds.

P.G. wishes to thank William (Bill) P. Blair for his kind hospitality in the Rowland Department of Physics \& Astronomy at the Johns Hopkins University.

M.H. acknowledges the support of the Spanish Ministry of Economy and Competitiveness (MINECO) under the grant FDPI-2013-16933 as well as the support of the Generalitat de Catalunya/CERCA programme.

S.C.W. acknowledges a visiting research fellowship at Liverpool John Moores University.

K.H. was supported by the project RVO:67985815.

V.A.R.M.R. acknowledges partial financial support from the Radboud Excellence Initiative, from Fundação para a Ciência e a Tecnologia (FCT) in the form of an exploratory project of reference IF/00498/2015, from the Center for Research \& Development in Mathematics and Applications (CIDMA) strategic project UID/MAT/04106/2013, and from Enabling Green E-science for the Square Kilometer Array Research Infrastructure (ENGAGE SKA), POCI-01-0145-FEDER022217, funded by Programa Operacional Competitividade e Internacionalização (COMPETE 2020) and FCT, Portugal.

This work has been supported in part by NSF grant AST1009566. Support for program \#14125 was provided by NASA through a grant from the Space Telescope Science Institute, which is operated by the Association of Universities for Research in Astronomy, Inc., under NASA contract NAS 5-26555.

Facilities: HST (WFC3), Keck:I (LRIS).

Software: calwf3 pipeline (v3.1.6; Dressel 2012), Dolphot (v2.0; Dolphin 2000), Drizzlepac (v2.0.2), IRAF (v2.16.1, Tody et al. 1993), PGPLOT (v5.2), Synspec/Tlusty (v202; Hubeny \& Lanz 1995), wfc3uv_ctereverse_parallel (v2015.07.22; Anderson et al. 2012). 


\section{ORCID iDs}

M. J. Darnley (1) https://orcid.org/0000-0003-0156-3377

R. Hounsell (1) https://orcid.org/0000-0002-0476-4206

P. Godon (1) https://orcid.org/0000-0002-4806-5319

D. A. Perley (1) https://orcid.org/0000-0001-8472-1996

B. F. Williams (1) https://orcid.org/0000-0002-7502-0597

S. C. Williams (i) https://orcid.org/0000-0002-4237-3994

V. A. R. M. Ribeiro (ํ) https://orcid.org/0000-0003-3617-4400

A. W. Shafter (1) https://orcid.org/0000-0002-1276-1486

M. M. Shara 나 https://orcid.org/0000-0003-0155-2539

\section{References}

Abramowicz, M. A., Czerny, B., Lasota, J. P., \& Szuszkiewicz, E. 1988, ApJ, 332,646

Anderson, J., MacKenty, J., Baggett, S., \& Noeske, K. 2012, The Efficacy of PostFlashing for Mitigating CTE-Losses in WFC3/UVIS Images (Baltimore, MD: STSci), http://www.stsci.edu/hst/wfc3/ins_performance/CTE/ ANDERSON_UVIS_POSTFLASH_EFFICACY.pdf

Ashall, C., Mazzali, P., Sasdelli, M., \& Prentice, S. J. 2016, MNRAS, 460, 3529

Barsukova, E., Fabrika, S., Hornoch, K., et al. 2011, ATel, 3725, 1

Bode, M. F., \& Evans, A. 2008, Classical Novae (2nd ed.; Cambridge: Cambridge Univ. Press)

Bode, M. F., Darnley, M. J., Beardmore, A. P., et al. 2016, ApJ, 818, 145

Bode, M. F., \& Kahn, F. D. 1985, MNRAS, 217, 205

Bode, M. F., O'Brien, T. J., Osborne, J. P., et al. 2006, ApJ, 652, 629

Cao, Y., Kasliwal, M. M., Neill, J. D., et al. 2012, ApJ, 752, 133

Dalcanton, J. J., Williams, B. F., Lang, D., et al. 2012, ApJS, 200, 18

Darnley, M. J., Henze, M., Bode, M. F., et al. 2016, ApJ, 833, 149

Darnley, M. J., Henze, M., Shafter, A. W., \& Kato, M. 2015a, ATel, 7964, 1

Darnley, M. J., Henze, M., Shafter, A. W., \& Kato, M. 2015b, ATel, 7965, 1

Darnley, M. J., Henze, M., Steele, I. A., et al. 2015c, A\&A, 580, A45

Darnley, M. J., Hounsell, R., Godon, P., et al. 2017, ApJ, 847, 35

Darnley, M. J., Hounsell, R. A., \& Bode, M. F. 2008, in ASP Conf. Ser. 401, RS Ophiuchi (2006) and the Recurrent Nova Phenomenon, ed. A. Evans et al. (San Francisco, CA: ASP), 203

Darnley, M. J., Ribeiro, V. A. R. M., Bode, M. F., Hounsell, R. A., \& Williams, R. P. 2012, ApJ, 746, 61

Darnley, M. J., Williams, S. C., Bode, M. F., et al. 2014, A\&A, 563, L9

Dolphin, A. E. 2000, PASP, 112, 1383

Dressel, L. 2012, Wide Field Camera 3 Instrument Handbook for Cycle 21 v. 5.0 (Baltimore, MD: STScI)

Ellis, G. L., Grayson, E. T., \& Bond, H. E. 1984, PASP, 96, 283

Evans, A., Bode, M. F., O’Brien, T. J., \& Darnley, M. J. (ed.) 2008, in ASP Conf. Ser. 401, RS Ophiuchi (2006) and the Recurrent Nova Phenomenon (San Francisco, CA: ASP), 1

Fujimoto, M. Y. 1982, ApJ, 257, 752

Godon, P. 1997, ApJ, 483, 882

Godon, P., Sion, E. M., Balman, S.., \& Blair, W. P. 2017, ApJ, 846, 52

Godon, P., Sion, E. M., Starrfield, S., et al. 2014, ApJL, 784, L33

Hachisu, I., \& Kato, M. 2006, ApJS, 167, 59

Hachisu, I., \& Kato, M. 2007, ApJ, 662, 552

Hachisu, I., Kato, M., Kiyota, S., et al. 2008, in ASP Conf. Ser. 401, RS Ophiuchi (2006) and the Recurrent Nova Phenomenon, ed. A. Evans et al. (San Francisco, CA: ASP), 206

Hachisu, I., Saio, H., \& Kato, M. 2016, ApJ, 824, 22

Henze, M., Darnley, M. J., Kabashima, F., et al. 2015a, A\&A, 582, L8 Henze, M., Darnley, M. J., Shafter, A. W., et al. 2015c, ATel, 7984, 1

Henze, M., Darnley, M. J., Williams, S. C., et al. 2017, ApJ, submitted Henze, M., Ness, J.-U., Darnley, M. J., et al. 2014, A\&A, 563, L8

Henze, M., Ness, J.-U., Darnley, M. J., et al. 2015b, A\&A, 580, A46

Hillman, Y., Prialnik, D., Kovetz, A., \& Shara, M. M. 2016, ApJ, 819, 168

Hillwig, T., Livio, M., \& Honeycutt, R. K. 2004, PASP, 116, 397

Hubeny, I. 1988, CoPhC, 52, 103

Hubeny, I., \& Lanz, T. 1995, ApJ, 439, 875

Hubeny, I., Lanz, T., \& Jeffrey, S. 1994, St. Andrews Univ. Newsletter on Analysis of Astronomical Spectra, 20, 30

Itagaki, K., Gao, X., Darnley, M. J., et al. 2016, ATel, 9848, 1

Kato, M., Saio, H., \& Hachisu, I. 2015, ApJ, 808, 52
Kato, M., Saio, H., \& Hachisu, I. 2017a, ApJ, 844, 143

Kato, M., Saio, H., \& Hachisu, I. 2017b, ApJ, 838, 153

Kato, M., Saio, H., Hachisu, I., \& Nomoto, K. 2014, ApJ, 793, 136

Kato, M., Saio, H., Henze, M., et al. 2016, ApJ, 830, 40

King, A. R. 1998, MNRAS, 296, L45

Knigge, C., King, A. R., \& Patterson, J. 2000, A\&A, 364, L75

Korotkiy, S., \& Elenin, L. 2011, Transient Object Followup Reports, Tech. Rep. TCP J00452884+4154095 (Cambridge, MA: CBAT), http://www. cbat.eps.harvard.edu/unconf/followups/J00452885+4154094.html

Krautter, J. 2002, in AIP Conf. Ser. 637, Classical Nova Explosions, ed M. Hernanz \& J. José (New York: AIP), 345

Livio, M. 1997, in ASP Conf. Ser. 121, IAU Colloq. 163: Accretion Phenomena and Related Out ows, ed. D. T. Wickramasinghe, G. V. Bicknell, \& L. Ferrario (San Francisco, CA: ASP), 845

Matthews, J. H., Knigge, C., Long, K. S., Sim, S. A., \& Higginbottom, N. 2015, MNRAS, 450, 3331

McCarthy, J. K., Cohen, J. G., Butcher, B., et al. 1998, Proc. SPIE, 3355, 81 Narayan, R., \& Yi, I. 1994, ApJL, 428, L13

Narayan, R., \& Yi, I. 1995, ApJ, 444, 231

Nishiyama, K., \& Kabashima, F. 2008, M31 (Apparent) Novae Page, Tech. Rep. (Cambridge, MA: CBAT), http://www.cbat.eps.harvard.edu/iau/CBAT_ M31.html\#2008-12a

Nishiyama, K., \& Kabashima, F. 2012, Transient Object Followup Reports, Tech. Rep. PNV J00452885+4154094 (Cambridge, MA: CBAT), http:// www.cbat.eps.harvard.edu/unconf/followups/J00452884+4154095.html

Nomoto, K. 1982, ApJ, 253, 798

Oke, J. B., Cohen, J. G., Carr, M., et al. 1995, PASP, 107, 375

Page, K. L., Osborne, J. P., Kuin, N. P. M., et al. 2015, MNRAS, 454, 3108

Pagnotta, A., Schaefer, B. E., Xiao, L., Collazzi, A. C., \& Kroll, P. 2009, AJ, 138, 1230

Perryman, M. A. C., Lindegren, L., Kovalevsky, J., et al. 1997, A\&A, 323 , L49

Pietrinferni, A., Cassisi, S., Salaris, M., \& Castelli, F. 2004, ApJ, 612, 168

Popham, R. 1997, ApJ, 478, 734

Popham, R., \& Narayan, R. 1995, ApJ, 442, 337

Poutanen, J., Lipunova, G., Fabrika, S., Butkevich, A. G., \& Abolmasov, P. 2007, MNRAS, 377, 1187

Pringle, J. E. 1977, MNRAS, 178, 195

Puebla, R. E., Diaz, M. P., \& Hubeny, I. 2007, AJ, 134, 1923

Rockosi, C., Stover, R., Kibrick, R., et al. 2010, Proc. SPIE, 7735, 77350R

Schaefer, B. E. 2010, ApJS, 187, 275

Shafter, A. W. 2017, ApJ, 834, 196

Shafter, A. W., Hornoch, K., Ciardullo, J. V. R., Darnley, M. J., \& Bode, M. F. 2012, ATel, 4503, 1

Shahbaz, T., \& Kuulkers, E. 1998, MNRAS, 295, L1

Shakura, N. I., \& Sunyaev, R. A. 1973, A\&A, 24, 337

Skrutskie, M. F., Cutri, R. M., Stiening, R., et al. 2006, AJ, 131, 1163

Starrfield, S. 2016, Handbook of Supernovae (Cham: Springer)

Starrfield, S., Iliadis, C., \& Hix, W. R. 2016, PASP, 128, 051001

Starrfield, S., Sparks, W. M., \& Truran, J. W. 1976, in IAU Symp. 73, Structure and Evolution of Close Binary Systems, ed. P. Eggleton, S. Mitton, \& J. Whelan (Cambridge: Cambridge Univ. Press), 155

Starrfield, S., Truran, J. W., Sparks, W. M., Krautter, J., \& MacDonald, J. 1990, in IAU Colloq. 122, Physics of Classical Novae, 369, ed. A. Cassatella \& R. Viotti (Berlin: Springer), 306

Tang, S., Bildsten, L., Wolf, W. M., et al. 2014, ApJ, 786, 61

Tang, S., Cao, Y., \& Kasliwal, M. M. 2013, ATel, 5607, 1

Tody, D. 1993, in ASP Conf. Ser. 52, Astronomical Data Analysis Software and Systems II, ed. R. J. Hanisch, R. J. V. Brissenden, \& J. Barnes (San Francisco, CA: ASP), 173

van Paradijs, J., \& McClintock, J. E. 1994, A\&A, 290, 133

Vaytet, N. M. H., O’Brien, T. J., Page, K. L., et al. 2011, ApJ, 740, 5

Vrtilek, S. D., Raymond, J. C., Garcia, M. R., et al. 1990, A\&A, 235, 162

Wade, R. A., \& Hubeny, I. 1998, ApJ, 509, 350

White, N. E., Giommi, P., Heise, J., Angelini, L., \& Fantasia, S. 1995, ApJL, $445, \mathrm{~L} 125$

Williams, B. F., Garcia, M. R., Kong, A. K. H., et al. 2004, ApJ, 609, 735

Williams, B. F., Lang, D., Dalcanton, J. J., et al. 2014a, ApJS, 215, 9

Williams, S. C., Darnley, M. J., Bode, M. F., Keen, A., \& Shafter, A. W. 2014b, ApJS, 213, 10

Worters, H. L., Eyres, S. P. S., Bromage, G. E., \& Osborne, J. P. 2007, MNRAS, 379, 1557

Woudt, P. A., \& Ribeiro, V. A. R. M. 2014, in ASP Conf. Ser. 490, Stella Novae: Past and Future Decades (San Francisco, CA: ASP), 1 